\title{
Systematic evaluation of cancer risk associated with rs2292832 in miR-149 and rs895819 in miR-27a: a comprehensive and updated meta-analysis
}

\author{
Yajing Feng ${ }^{1}$, Fujiao Duan' ${ }^{2}$ Chunhua Song ${ }^{3}$, Xia Zhao' ${ }^{2}$, Liping Dai ${ }^{3}$, Shuli Cui ${ }^{4}$ \\ ${ }^{1}$ Department of Infection Management, The First Affiliated Hospital of Zhengzhou University, Zhengzhou, 450052, Henan, \\ P.R.China \\ ${ }^{2}$ Department of Hospital Infection Management, Affiliated Cancer Hospital of Zhengzhou University, Henan Cancer Hospital, \\ Zhengzhou 450008, Henan, P.R.China \\ ${ }^{3}$ Department of Epidemiology, College of Public Health, Zhengzhou University, Zhengzhou 450001, Henan, P.R.China \\ ${ }^{4}$ College of Professional Study, Northeastern University, Boston, 02215 Massachusetts, USA \\ Correspondence to: Yajing Feng, e-mail: grglfyj@163.com \\ Fujiao Duan, e-mail: fjduan@yeah.net
}

Keywords: miR-149, miR-27a, cancer, susceptibility, systematic evaluation

Received: November 05, $2015 \quad$ Accepted: February 24, 2016

Published: March 12, 2016

\section{ABSTRACT}

The aim of this study is to provide a precise quantification for the association between miR-149 T > C (rs2292832) and miR-27a A > G (rs895819) and the risk of cancer. We conducted a systematic literature review and evaluated the quality of included studies based on Newcastle-Ottawa Scale (NOS). Pooled odds ratios (ORs) and corresponding $95 \%$ confidence intervals ( $95 \%$ CIs) were calculated to assess the strengths of the associations. We identified 40 studies for pooled analyses. Overall, the results demonstrated that the rs2292832 polymorphism was subtly decrease the risk of breast cancer (CT + CC vs TT: OR $=0.83,95 \%$ CI: $0.70-0.98, P=0.03 ;$ CC vs CT + TT: $O R=0.80,95 \% \mathrm{CI}: 0.68-0.93, P=0.00)$, and the rs895819 polymorphism was associated with significantly increased cancer risk in the Asian population (AG + GG vs $A A: O R=1.24,95 \% C I: 1.03-1.50, P=0.02$ ) and in colorectal cancer subgroup (GG vs $A A:$ OR $=1.45,95 \%$ CI: $1.10-1.92, P=0.00$; $A G+$ GG vs $A A:$ OR $=1.35,95 \%$ CI: 1.15-1.58, $P=0.00$; GG vs AG + AA: OR = 1.36, 95\% CI: 1.04-1.77, $P=0.02$ ). In addition, a subtly decreased risk was observed in the Caucasian population and in breast cancer subgroup. In conclusion, the rs 2292832 polymorphism was significantly associated with increased breast cancer risk, and the rs895819 polymorphism contributes to the susceptibility of colorectal and breast cancer.

\section{INTRODUCTION}

MicroRNAs (miRNAs) are a group of short noncoding RNAs of about 22 nucleotides which are involved in diverse physiological and developmental processes by controlling the gene expression of target mRNAs $[1,2]$. Accumulating evidence has shown that miRNAs regulate the expression of roughly $10-30 \%$ of the all human genes through post-transcriptional mechanisms [3], contributing to excessive physiologic and pathologic conditions, including cell differentiation, apoptosis, development, and deregulation of these processes play critical roles in carcinogenesis [4].
Single nucleotide polymorphisms (SNPs) represent the most common genetic variation in human genome. SNPs in miRNA genes are regarded to affect function by three ways: first, through the transcription of the primary transcript; second, through pri-miRNA and pre-miRNA processing; and third, through effects on miRNA-miRNA interactions [5]. Recently, several studies have demonstrated that some SNPs present in the miRNA genes $[6,7]$, which can alter miRNA expression and/or maturation and be associated with the development and progression of cancer [8]. Thus, SNPs in miRNAs may influence susceptibility to malignant tumors. The miR-149 $\mathrm{T}>\mathrm{C}(\mathrm{rs} 2292832)$ and miR-27a A > G (rs895819) were 
studied in diverse cancers. Research results about two sites were inconsistent $[9,10]$, this discrepancy maybe partially attributed to the heterogeneity of the cancer subtype, small sample size, and ethnicity of the patients.

To further determine whether there is an association of the rs2292832 and rs895819 in the miRNA genes with the risk for developing cancer, a comprehensive review and analysis of published data from different studies is needed. In this study, we performed a meta-analysis on all eligible case-control studies to drive a more powerful estimation of the association of rs2292832 and rs895819 SNP with cancer risks.

\section{RESULTS}

\section{Study characteristics}

The search process and the final selection of relevant studies are shown in Figure 1, A comprehensive literature search yielded 348 potentially relevant published articles. After further identification and screening individual study, 43 articles (49 studies) [11-53] underwent full-text assessment, and 6 articles (10 studies, not including one site according to HWE) $[14,17$, $19,20,35,42]$ were excluded due to inconsistently with HWE. Finally, 37 articles (40 studies) [11-13, 15, 16, 18, 21-34, 36-41, 43-53] were conducted in quantitative synthesis.

Characteristics of included studies are presented in Table 1. A total of 39 eligible studies met the prespecified inclusion criteria, in which two articles $[24,52]$ included two tumor types respectively, and one article included [23] rs2292832 and rs895819. As for rs2292832, involving 9,994 cases and 10,757 controls were ultimately analyzed from 21 studies (20 articles) [11-13, 15, 16, 18, 21-34], and 19 studies (17 articles) [23, 36-41, 43-53] involving 7,800 cases and 9,060 controls for rs895819.

All studies were case-control studies, including 40 studies on 10 breast cancer, 7 gastric cancer, 7 colorectal cancer, 4 lung cancer, and 12 on other cancer types. There were 28 studies of Asian descendent, 11 of Caucasian descendent. A classic PCR-RFLP assay was used in 17 out of 40 studies, the other molecular genotyping methods, such as Taqman, MassARRAY, and HRM, were used in other studies. 32 studies were randomly repeated a portion of samples as quality control while genotyping.

\section{Quality assessment}

According to the NOS for quality of case-control, the study-specific quality scores are summarized in Table 2. A star system of the NOS (range, 0-9 scores) has been developed for the evaluation, and the quality scores ranged from 4 to 8 . The average scores of case-control studies were 6.49 .

\section{Quantitative data synthesis}

For all of control subjects included in this study, the frequencies of risk $\mathrm{C}$ allele in rs2292832 for Caucasians and Asians were 33.66\% (Mean \pm SEM, 33.66\% \pm $2.18 \%$ ) and 50.20\% (Mean \pm SEM, 50.20\% $\pm 12.34 \%$ ) (Figure 2A). The frequencies of risk $\mathrm{G}$ allele in rs 895819 for Caucasians and Asians were 30.78\% (Mean \pm SEM, $30.78 \% \pm 2.04 \%$ ) and 29.63\% (Mean \pm SEM, 29.63\% $\pm 1.45 \%$ ) (Figure $2 \mathrm{~B}$ ). The frequencies of risk $\mathrm{C}$ allele in rs2292832 varied greatly among different control populations $(P=0.00)$.

For the rs2292832 polymorphism, no significant risk association was observed in the overall pooled analysis (Table 3, Figure 3). When grouped by the cancer types, significant associations were found in breast cancer $(\mathrm{CT}+\mathrm{CC}$ vs TT: $\mathrm{OR}=0.83,95 \% \mathrm{CI}: 0.70-0.98, P=0.03$; CC vs CT + TT: OR $=0.80,95 \%$ CI: $0.68-0.93, P=0.00$ ) (Table 4).

For the rs895819 polymorphism, we failed to find any associations between rs895819 polymorphism and cancer risk (Table 3, Figure 4). In the subgroup analysis by ethnicity, statistically significantly reduced cancer risks were found among Asian for dominant contrast $(\mathrm{AG}+\mathrm{GG}$ vs $\mathrm{AA}: \quad \mathrm{OR}=1.24,95 \% \mathrm{CI}: 1.03-1.50$, $P=0.02$ ) (Table 5). In contrast, a subtly decreased risk was observed in the Caucasian population $(\mathrm{G}$ vs A: $\mathrm{OR}=0.92$, 95\% CI: $0.85-0.99, P=0.03$; AG vs AA: OR $=0.92$, 95\% CI: $0.85-0.99, P=0.00$ ) (Table 5). Subgroup analysis by cancer types revealed a decreased risk in breast cancer (G vs A: OR = 0.92, 95\% CI: 0.86-0.99, $P=0.03$; AG vs AA: $\mathrm{OR}=0.83,95 \% \mathrm{CI}: 0.75-0.92, P<0.01 ; \mathrm{AG}+\mathrm{GG}$ vs $\mathrm{AA}: \mathrm{OR}=0.88,95 \% \mathrm{CI}: 0.80-0.97, P=0.01)$, whereas a significantly increased risk was observed in colorectal cancer (GG vs AA: OR $=1.45,95 \% \mathrm{CI}: 1.10-1.92$, $P<0.01 ; \mathrm{AG}+\mathrm{GG}$ vs AA: $\mathrm{OR}=1.35,95 \% \mathrm{CI}: 1.15-1.58$, $P<0.01 ; \mathrm{GG}$ vs $\mathrm{AG}+\mathrm{AA}: \mathrm{OR}=1.36,95 \% \mathrm{CI}: 1.04-1.77$, $P=0.02)$ (Table 5).

\section{Test of heterogeneity}

In the overall pooled analysis, the results showed that both rs2292832 and rs895819 had heterogeneity in part of genotype with $P$ value less than 0.05 . Therefore, we analyzed the summary ORs with random-effect models if the heterogeneity existed. Fixed-effect models were used to analyze the summary odds ratios for the rest. Subsequently, meta regression in Stata12.0 was used to assess the source of heterogeneity for rs2292832 and rs895819, including publication year, ethnicity (Asians, Caucasians), cancer type, matched controls (yes or not), language (English or Chinese), source of control (hospital or population), assay, sample size (300 as the boundary) and quality control (with or without). It was detected that the systemic results were not altered by these characteristics (Table 6). 


\section{Evaluation of publication bias}

Begg's funnel plot and Egger's test (Table 7) were performed to assess the publication bias of the currently available literature. The shape of the funnel plots did not reveal any evidence of obvious asymmetry in all comparison models (Figure 5 and Figure 6).

\section{Sensitivity analysis}

A single study included in the meta-analysis was deleted each time to reflect the influence of the individual data set to the pooled ORs, and the corresponding pooled ORs were not materially changed (data not shown).

\section{DISCUSSION}

In the present study, an association between the two common SNPs in microRNAs (rs2292832 and rs895819) and cancer risk was evaluated by the pooled results from 40 published studies. The results demonstrated that the rs2292832 was associated with a significantly reduced risk for developing cancer in the breast cancer (dominant and recessive model), and for the rs895819 G allele, $\mathrm{AG}$ genotype and dominant model were associated with a decreased risk for Caucasian population and breast cancer, in contrast, a subtly increased risk was observed in a Asian population (dominant model) and colorectal cancer (GG genotype, dominant model and recessive model).

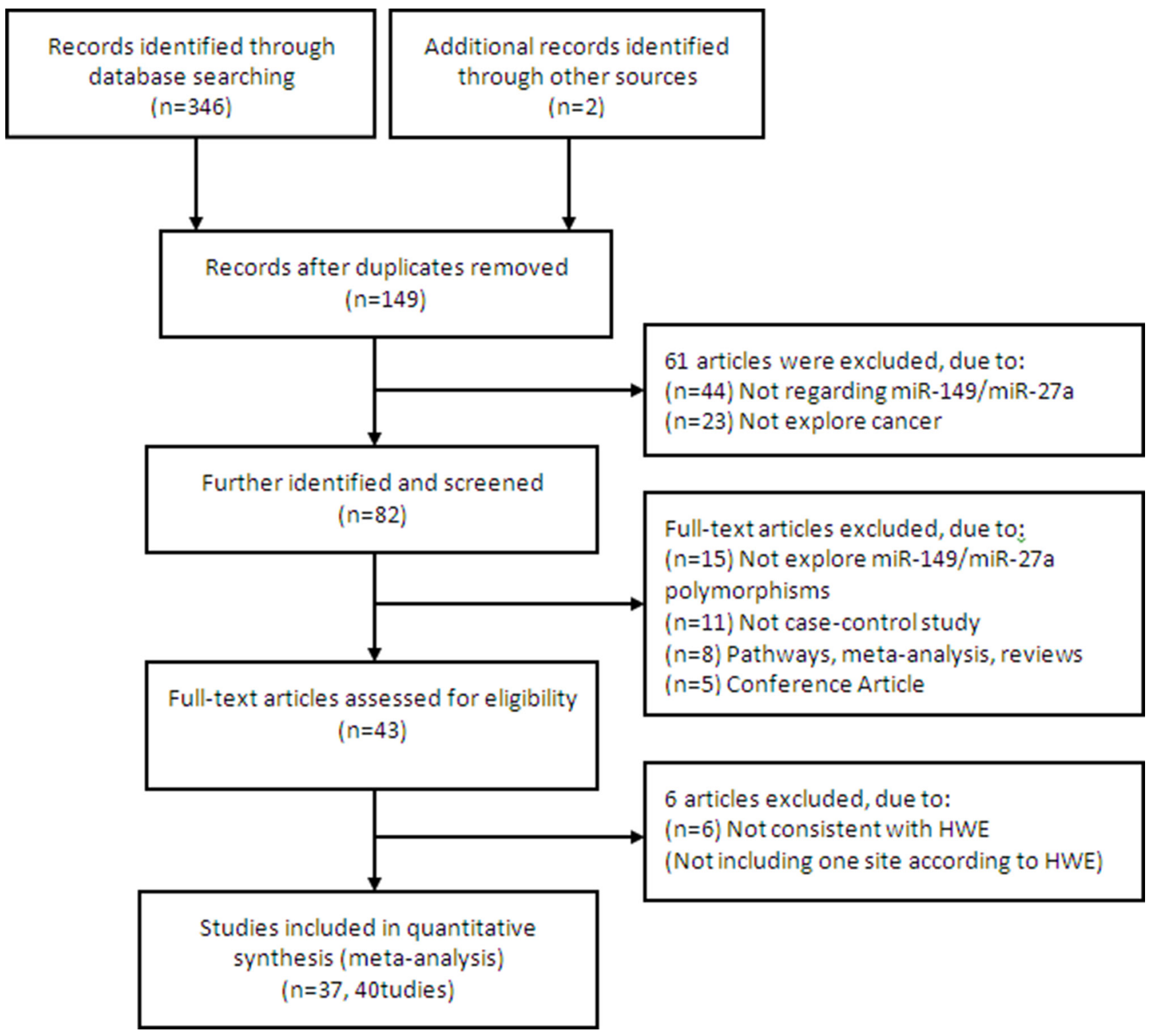

Figure 1: Flow chart of literature search and study selection. 
Table 1: Main characteristics of included studies

\begin{tabular}{|c|c|c|c|c|c|c|c|c|c|c|}
\hline \multirow[t]{2}{*}{ First author } & \multirow[t]{2}{*}{ Year } & \multirow[t]{2}{*}{ Ethnicity } & \multirow{2}{*}{$\begin{array}{l}\text { Cancer } \\
\text { type }\end{array}$} & \multirow{2}{*}{$\begin{array}{c}\text { Source of } \\
\text { control }\end{array}$} & \multirow[t]{2}{*}{ Genotyping } & \multirow{2}{*}{$\begin{array}{c}\text { Match }^{\mathbf{a}} \\
\mathrm{Y} / \mathrm{N}\end{array}$} & \multirow{2}{*}{$\begin{array}{c}\text { Sample size } \\
\text { Case/ } \\
\text { Control }\end{array}$} & \multicolumn{2}{|c|}{$P_{\text {HWE }}$} & \multirow{2}{*}{$\begin{array}{l}\text { Quality } \\
\text { control }\end{array}$} \\
\hline & & & & & & & & rs2292832 & rs895819 & \\
\hline He BS [11] & 2015 & Asian & $\begin{array}{l}\text { Breast } \\
\text { cancer }\end{array}$ & Population & MassARRAY & $\mathrm{Y}$ & $450 / 450$ & 0.13 & & $\mathrm{Y}$ \\
\hline Du ML [12] & 2014 & Asian & $\begin{array}{c}\text { Renal cell } \\
\text { cancer }\end{array}$ & Population & TaqMan & $\mathrm{Y}$ & $355 / 362$ & 0.46 & & $\mathrm{Y}$ \\
\hline $\begin{array}{l}\text { Dikeakos } \\
\mathrm{P}[13]\end{array}$ & 2014 & Caucasian & $\begin{array}{l}\text { Gastric } \\
\text { cancer }\end{array}$ & Hospital & PCR-RFLP & $\mathrm{Y}$ & $163 / 480$ & 0.45 & & $\mathrm{Y}$ \\
\hline $\mathrm{Pu} J Y[14]$ & 2014 & Asian & $\begin{array}{l}\text { Gastric } \\
\text { cancer }\end{array}$ & Hospital & PCR-RFLP & $\mathrm{N}$ & $220 / 530$ & $<0.01$ & & $\mathrm{Y}$ \\
\hline Wei WJ [15] & 2014 & Asian & PTC & Population & MassARRAY & $\mathrm{Y}$ & $838 / 1006$ & 0.73 & & $\mathrm{Y}$ \\
\hline $\begin{array}{l}\text { Wang } \\
\mathrm{R}[16]\end{array}$ & 2014 & Asian & $\mathrm{HCC}$ & Population & MassARRAY & $\mathrm{N}$ & $944 / 984$ & 0.86 & & $\mathrm{~N}$ \\
\hline Wu RR [17] & 2014 & Asian & $\begin{array}{c}\text { Colorectal } \\
\text { Cancer }\end{array}$ & Hospital & ASA & $\mathrm{N}$ & $175 / 300$ & $<0.01$ & 0.02 & $\mathrm{Y}$ \\
\hline $\begin{array}{l}\text { Huang } \\
\text { GL [18] }\end{array}$ & 2013 & Asian & NPC & Population & PCR-RFLP & $\mathrm{N}$ & $158 / 242$ & 0.72 & & Y \\
\hline $\begin{array}{l}\text { Chu } \\
\text { YH [19] }\end{array}$ & 2013 & Asian & $\mathrm{HCC}$ & Population & PCR-RFLP & $\mathrm{N}$ & $188 / 337$ & $<0.01$ & & $\mathrm{Y}$ \\
\hline Lv M [20] & 2013 & Asian & $\begin{array}{c}\text { Colorectal } \\
\text { cancer }\end{array}$ & Population & PCR-RFLP & $\mathrm{N}$ & $353 / 540$ & $<0.01$ & & $\mathrm{Y}$ \\
\hline $\begin{array}{l}\text { Song } \\
\mathrm{XC}[21]\end{array}$ & 2013 & Caucasian & OSCC & Population & PCR-RFLP & $\mathrm{Y}$ & $325 / 335$ & 0.99 & & $\mathrm{Y}$ \\
\hline Tu HF [22] & 2012 & Asian & HNSCC & Hospital & PCR-RFLP & $\mathrm{N}$ & $122 / 273$ & 0.27 & & NA \\
\hline $\begin{array}{l}\text { Zhang } \\
\text { M [23] }\end{array}$ & 2012 & Asian & $\begin{array}{l}\text { Breast } \\
\text { Cancer }\end{array}$ & Population & PCR-RFLP & $\mathrm{Y}$ & $252 / 248$ & 0.21 & 0.12 & $\mathrm{Y}$ \\
\hline $\begin{array}{l}\text { Zhang } \\
\text { MW(C) [24] }\end{array}$ & 2012 & Asian & $\begin{array}{c}\text { Colorectal } \\
\text { Cancer }\end{array}$ & Population & PCR-RFLP & $\mathrm{Y}$ & $443 / 435$ & 0.43 & & $\mathrm{Y}$ \\
\hline $\begin{array}{l}\text { Zhang } \\
\text { MW(G) [24] }\end{array}$ & 2012 & Asian & $\begin{array}{l}\text { Gastric } \\
\text { Cancer } \\
\end{array}$ & Population & PCR-RFLP & $\mathrm{Y}$ & $274 / 269$ & 0.70 & & $\mathrm{Y}$ \\
\hline $\begin{array}{l}\text { Min } \\
\text { KT [25] }\end{array}$ & 2012 & Asian & $\begin{array}{c}\text { Colorectal } \\
\text { Cancer }\end{array}$ & Population & PCR-RFLP & $\mathrm{N}$ & $446 / 502$ & 0.62 & & $\mathrm{Y}$ \\
\hline $\begin{array}{l}\text { Ahn } \\
\text { DH [26] }\end{array}$ & 2012 & Asian & $\begin{array}{l}\text { Gastric } \\
\text { Cancer }\end{array}$ & Population & PCR-RFLP & $\mathrm{N}$ & $461 / 447$ & 0.98 & & $\mathrm{Y}$ \\
\hline $\begin{array}{l}\text { Kim } \\
\text { WH [27] }\end{array}$ & 2012 & Asian & $\mathrm{HCC}$ & Population & PCR-RFLP & $\mathrm{N}$ & $159 / 201$ & 0.34 & & $\mathrm{Y}$ \\
\hline Vinci S [28] & 2013 & Caucasian & $\begin{array}{c}\text { Colorectal } \\
\text { Cancer }\end{array}$ & Population & HRM & $\mathrm{Y}$ & $160 / 178$ & 0.91 & & Y \\
\hline Vinci S [29] & 2011 & Caucasian & $\begin{array}{c}\text { Lung } \\
\text { Cancer }\end{array}$ & Population & HRM & $\mathrm{Y}$ & $101 / 129$ & 0.97 & & $\mathrm{Y}$ \\
\hline Li PY [30] & 2011 & Asian & NPC & Hospital & TaqMan & $\mathrm{Y}$ & $791 / 1016$ & 0.49 & & NA \\
\hline $\begin{array}{l}\text { Zhang } \\
\text { MW [31] }\end{array}$ & 2011 & Asian & $\begin{array}{c}\text { Lung } \\
\text { Cancer }\end{array}$ & Population & PCR-RFLP & $\mathrm{Y}$ & $232 / 231$ & 0.12 & & $\mathrm{Y}$ \\
\hline Liu ZS [32] & 2010 & Caucasian & HNSSC & Population & PCR-RFLP & $\mathrm{Y}$ & $1109 / 1130$ & 0.72 & & $\mathrm{Y}$ \\
\hline Tian T [33] & 2009 & Asian & $\begin{array}{c}\text { Lung } \\
\text { Cancer }\end{array}$ & Population & PCR-RFLP & $\mathrm{Y}$ & $1058 / 1035$ & 0.86 & & $\mathrm{Y}$ \\
\hline $\begin{array}{l}\text { Wang } \\
\text { ZW [34] }\end{array}$ & 2009 & Asian & $\begin{array}{l}\text { Breast } \\
\text { Cancer }\end{array}$ & Population & PCR-RFLP & $\mathrm{Y}$ & $1009 / 1093$ & 0.16 & & $\mathrm{Y}$ \\
\hline Ma JY [35] & 2015 & Asian & $\mathrm{NSCC}$ & Population & TaqMan & $\mathrm{Y}$ & $542 / 557$ & & 0.02 & $\mathrm{Y}$ \\
\hline Qi P [36] & 2015 & Asian & $\begin{array}{l}\text { Breast } \\
\text { cancer }\end{array}$ & Population & TaqMan & $\mathrm{Y}$ & $321 / 290$ & & 0.69 & $\mathrm{~N}$ \\
\hline Yin $\mathrm{ZH}$ [37] & 2015 & Asian & $\begin{array}{l}\text { Lung } \\
\text { Cancer }\end{array}$ & Hospital & TaqMan & $\mathrm{Y}$ & $258 / 310$ & & 0.70 & $\mathrm{Y}$ \\
\hline
\end{tabular}




\begin{tabular}{|c|c|c|c|c|c|c|c|c|c|c|}
\hline Cao Y [38] & 2014 & Asian & $\begin{array}{c}\text { Colorectal } \\
\text { cancer }\end{array}$ & Population & PCR-RFLP & $\mathrm{Y}$ & $254 / 238$ & & 0.09 & $\mathrm{Y}$ \\
\hline \begin{tabular}{|l|} 
Kupcinskas J \\
(C) [39]
\end{tabular} & 2014 & Caucasian & $\begin{array}{c}\text { Colorectal } \\
\text { cancer }\end{array}$ & Hospital & TaqMan & $\mathrm{N}$ & $193 / 428$ & & 0.24 & $\mathrm{Y}$ \\
\hline \begin{tabular}{|l|} 
Kupcinskas J \\
$(\mathrm{G})[40]$
\end{tabular} & 2014 & Caucasian & $\begin{array}{l}\text { Gastric } \\
\text { cancer }\end{array}$ & Hospital & TaqMan & $\mathrm{N}$ & $363 / 351$ & & 0.15 & $\mathrm{Y}$ \\
\hline Song B [41] & 2014 & Asian & $\begin{array}{l}\text { Gastric } \\
\text { cancer }\end{array}$ & Population & TaqMan & $\mathrm{Y}$ & $278 / 278$ & & 0.11 & $\mathrm{Y}$ \\
\hline \begin{tabular}{|l} 
Wang \\
ZQ [42] \\
\end{tabular} & 2014 & Asian & $\begin{array}{c}\text { Colorectal } \\
\text { cancer }\end{array}$ & Hospital & TaqMan & $\mathrm{N}$ & $205 / 455$ & & $<0.01$ & $\mathrm{Y}$ \\
\hline \begin{tabular}{|l} 
Zhang \\
JJ [43]
\end{tabular} & 2014 & Asian & ESCC & Population & SNaPshot & $\mathrm{Y}$ & $1109 / 1275$ & & 0.23 & $\mathrm{Y}$ \\
\hline \begin{tabular}{|l|} 
Zhang \\
N [44] \\
\end{tabular} & 2013 & Asian & $\begin{array}{l}\text { Breast } \\
\text { cancer }\end{array}$ & Population & TaqMan & $\mathrm{Y}$ & $264 / 255$ & & 0.45 & $\mathrm{~N}$ \\
\hline \begin{tabular}{|l|} 
Catucci \\
I [45]
\end{tabular} & 2012 & Caucasian & $\begin{array}{l}\text { Breast } \\
\text { Cancer }\end{array}$ & Hospital & TaqMan & $\mathrm{Y}$ & $1,025 / 1,593$ & & 0.051 & $\mathrm{Y}$ \\
\hline \begin{tabular}{|l|} 
Hezova \\
$\mathrm{R}[46]$ \\
\end{tabular} & 2012 & Caucasian & $\begin{array}{c}\text { Colorectal } \\
\text { Cancer }\end{array}$ & Population & TaqMan & $\mathrm{Y}$ & $197 / 202$ & & 0.87 & NA \\
\hline Shi DN [47] & 2012 & Asian & $\begin{array}{c}\text { Renal Cell } \\
\text { Cance }\end{array}$ & Population & TaqMan & $\mathrm{Y}$ & $594 / 600$ & & 0.37 & $\mathrm{Y}$ \\
\hline \begin{tabular}{|l|} 
Zhang \\
MW [48]
\end{tabular} & 2012 & Asian & $\begin{array}{c}\text { Colorectal } \\
\text { Cancer }\end{array}$ & Population & PCR-RFLP & $\mathrm{Y}$ & $463 / 468$ & & 0.35 & $\mathrm{Y}$ \\
\hline Zhou Y [49] & 2012 & Asian & $\begin{array}{l}\text { Gastric } \\
\text { cancer }\end{array}$ & Hospital & MassARRAY & $\mathrm{Y}$ & $311 / 425$ & & 0.94 & Y \\
\hline \begin{tabular}{|l|} 
Zhang \\
$\mathrm{P}[50]$ \\
\end{tabular} & 2011 & Asian & $\begin{array}{l}\text { Breast } \\
\text { Cancer }\end{array}$ & Population & MassARRAY & $\mathrm{Y}$ & $384 / 192$ & $<0.01$ & 0.61 & $\mathrm{Y}$ \\
\hline $\begin{array}{l}\text { Sun } \\
\text { QM [51] }\end{array}$ & 2010 & Asian & $\begin{array}{l}\text { Gastric } \\
\text { cancer }\end{array}$ & Hospital & PCR-RFLP & $\mathrm{Y}$ & $304 / 304$ & & 0.053 & Y \\
\hline \begin{tabular}{|l|} 
Kontorovich \\
T(B) [57] \\
\end{tabular} & 2010 & Caucasian & $\begin{array}{l}\text { Breast } \\
\text { cancer }\end{array}$ & Population & iPLEX & $\mathrm{N}$ & $86 / 106$ & $<0.01$ & 0.37 & $\mathrm{Y}$ \\
\hline \begin{tabular}{|l|} 
Kontorovich \\
$\mathrm{T}(\mathrm{O})[52]$ \\
\end{tabular} & 2010 & Caucasian & $\begin{array}{c}\text { Ovarian } \\
\text { cancer }\end{array}$ & Population & iPLEX & $\mathrm{N}$ & $34 / 106$ & $<0.01$ & 0.37 & $\mathrm{Y}$ \\
\hline $\begin{array}{l}\text { Yang } \\
\text { RX [53] }\end{array}$ & 2010 & Caucasian & $\begin{array}{l}\text { Breast } \\
\text { cancer }\end{array}$ & Population & TaqMan & $\mathrm{Y}$ & $1189 / 1416$ & & 0.14 & Y \\
\hline
\end{tabular}

${ }^{a}$ Match, controls and cases were matched on age and gender; ASA, allele-specific amplification; OSCC, oral squamous cell carcinoma; HNSCC, head and neck squamous cell carcinoma; HCC, hepatic cell carcinoma; NPC, Nasopharyngeal Carcinoma; NSCC, Non small cell Lung cancer; PTC, Papillary Thyroid Cancer.

Thus far, for the rs2292832, no significant association was observed in overall pooled results $[54,55]$. In contrast to the published results, this study revealed the different association between rs2292832 polymorphism and breast cancer risk. This suggests that the molecular mechanisms underlying the genetic associations of miRNA-SNPs with cancer are complex and vary by cancer site. Considering the influence of the $\mathrm{T}$ allele in rs2292832 might be masked by the presence of other as-yet unidentified causal genes involved in cancer development on this polymorphism [56], our results should be interpreted with caution, and more studies will need to be analyzed to confirm the results.

The rs895819 is well recognized to be involved in the pathogenesis, metastasis, and invasion of multiple cancer types, by functioning as an oncogene via complex mechanisms [57-59]. The rs895819, as an oncomiR, exhibited its oncogenic activity through regulating target genes $[60,61]$. It means that down-regulation of miR-27a may contribute to decreased cancer risk through up-regulating the targets. Although the binding of the mature miRNA to target mRNAs was not influenced by the rs895819 [62], some published studies had demonstrated that polymorphisms in premiRNAs could influence the expression of their mature forms, as well as were involved in the binding of some nuclear factors in miRNA processing [63]. Therefore, we presumed that rs895819 affected the processing or/and expression of miR-27a, which resulted in down-regulation of miR-27a. The presumption was supported by our findings in breast cancer subgroup.

This comprehensive and updated meta-analysis further support the rs895819 G allele was associated with a decreased risk for breast cancer, whereas a subtly 
Table 2: Quality assessment of included studies based on the newcastle-ottawa scale

\begin{tabular}{|c|c|c|c|c|c|c|c|c|c|}
\hline \multirow[t]{2}{*}{ Study } & $\begin{array}{l}\text { Selection } \\
\text { (score) }\end{array}$ & & & & $\begin{array}{c}\text { Comparability } \\
\text { (score) }\end{array}$ & $\begin{array}{l}\text { Exposure } \\
\text { (score) }\end{array}$ & & & $\begin{array}{r}\text { Total } \\
\text { score }^{\mathrm{b}}\end{array}$ \\
\hline & $\begin{array}{l}\text { Adequate } \\
\text { definition } \\
\text { of patient } \\
\text { case }\end{array}$ & $\begin{array}{c}\text { Representativeness } \\
\text { of patients cases }\end{array}$ & $\begin{array}{c}\text { Selection } \\
\text { of controls }\end{array}$ & $\begin{array}{l}\text { Definition } \\
\text { of control }\end{array}$ & $\begin{array}{l}\text { Control for } \\
\text { important } \\
\text { factor or } \\
\text { additional } \\
\text { factor }\end{array}$ & $\begin{array}{c}\text { Ascertainment } \\
\text { of exposure } \\
\text { (blinding) }\end{array}$ & $\begin{array}{c}\text { Same } \\
\text { method of } \\
\text { ascertainment } \\
\text { for } \\
\text { participants }\end{array}$ & $\begin{array}{l}\text { Non- } \\
\text { response } \\
\text { rate }^{\mathrm{a}}\end{array}$ & \\
\hline He BS [11] & 1 & 1 & 1 & 1 & 2 & 0 & 1 & 0 & 7 \\
\hline Du ML [12] & 1 & 1 & 1 & 1 & 2 & 0 & 1 & 0 & 7 \\
\hline \begin{tabular}{|l|} 
Dikeakos \\
P [13]
\end{tabular} & 1 & 1 & 0 & 1 & 2 & 0 & 1 & 1 & 7 \\
\hline Wei WJ [15] & 1 & 1 & 1 & 1 & 2 & 0 & 1 & 0 & 7 \\
\hline Wang R [16] & 1 & 1 & 0 & 1 & 1 & 0 & 1 & 0 & 5 \\
\hline \begin{tabular}{|l} 
Huang \\
GL [18]
\end{tabular} & 1 & 1 & 1 & 1 & 2 & 0 & 1 & 0 & 7 \\
\hline \begin{tabular}{|l|} 
Song XC \\
{$[21]$} \\
\end{tabular} & 1 & 1 & 1 & 1 & 2 & 0 & 1 & 1 & 8 \\
\hline Tu HF [22] & 1 & 1 & 0 & 1 & 2 & 0 & 1 & 0 & 6 \\
\hline $\begin{array}{l}\text { Zhang M } \\
{[23]}\end{array}$ & 1 & 1 & 1 & 1 & 2 & 0 & 1 & 0 & 7 \\
\hline \begin{tabular}{|l|} 
Zhang \\
MW [24] \\
\end{tabular} & 1 & 1 & 1 & 1 & 2 & 0 & 1 & 0 & 7 \\
\hline Min KT [25] & 1 & 1 & 1 & 1 & 2 & 0 & 1 & 0 & 7 \\
\hline Aho DH [26] & 1 & 1 & 1 & 1 & 2 & 0 & 1 & 1 & 8 \\
\hline Kim WH [27] & 1 & 1 & 1 & 1 & 2 & 0 & 1 & 0 & 7 \\
\hline Vinci S [28] & 1 & 1 & 1 & 1 & 1 & 0 & 1 & 0 & 6 \\
\hline Vinci S [29] & 1 & 1 & 0 & 1 & 2 & 0 & 1 & 0 & 7 \\
\hline Li PY [30] & 1 & 1 & 0 & 1 & 2 & 0 & 1 & 0 & 6 \\
\hline \begin{tabular}{|l} 
Zhang \\
MW [31]
\end{tabular} & 1 & 1 & 1 & 1 & 2 & 0 & 1 & 0 & 7 \\
\hline Liu ZS [32] & 1 & 1 & 1 & 1 & 2 & 0 & 1 & 1 & 8 \\
\hline Tian T [33] & 1 & 1 & 1 & 1 & 2 & 0 & 1 & 0 & 7 \\
\hline \begin{tabular}{|l} 
Wang \\
ZW [34]
\end{tabular} & 1 & 1 & 1 & 1 & 1 & 0 & 1 & 0 & 6 \\
\hline Qi P [36] & 1 & 1 & 1 & 1 & 2 & 0 & 1 & 0 & 7 \\
\hline Yin $\mathrm{ZH}$ [37] & 1 & 1 & 0 & 1 & 2 & 0 & 1 & 0 & 6 \\
\hline Cao Y [38] & 1 & 1 & 1 & 1 & 2 & 0 & 1 & 0 & 7 \\
\hline $\begin{array}{l}\text { Kupcinskas } \\
\text { J (C) [39] }\end{array}$ & 1 & 1 & 0 & 1 & 0 & 0 & 1 & 0 & 4 \\
\hline $\begin{array}{l}\text { Kupcinskas } \\
\mathrm{J}(\mathrm{G})[40]\end{array}$ & 1 & 1 & 0 & 1 & 2 & 0 & 1 & 0 & 6 \\
\hline Song B [41] & 1 & 1 & 1 & 1 & 2 & 0 & 1 & 0 & 7 \\
\hline Zhang JJ [42] & 1 & 1 & 1 & 1 & 2 & 0 & 1 & 0 & 7 \\
\hline Zhang N [43] & 1 & 1 & 1 & 1 & 2 & 0 & 1 & 0 & 7 \\
\hline Catucci I [44] & 1 & 1 & 0 & 1 & 1 & 0 & 1 & 0 & 5 \\
\hline \begin{tabular}{|l} 
Hezova \\
$\mathrm{R}[45]$ \\
\end{tabular} & 1 & 1 & 1 & 1 & 1 & 0 & 1 & 0 & 6 \\
\hline Shi DN [46] & 1 & 1 & 1 & 1 & 2 & 0 & 1 & 0 & 7 \\
\hline \begin{tabular}{|l} 
Zhang \\
MW [47]
\end{tabular} & 1 & 1 & 1 & 1 & 0 & 0 & 1 & 0 & 5 \\
\hline
\end{tabular}




\begin{tabular}{|c|c|c|c|c|c|c|c|c|c|}
\hline Zhou Y [49] & 1 & 1 & 0 & 1 & 1 & 0 & 1 & 0 & 5 \\
\hline Zhang P [50] & 1 & 1 & 1 & 1 & 1 & 0 & 1 & 0 & 6 \\
\hline Sun QM [51] & 1 & 1 & 0 & 1 & 2 & 0 & 1 & 0 & 6 \\
\hline $\begin{array}{l}\text { Kontorovich } \\
\mathrm{T}[52]\end{array}$ & 1 & 1 & 1 & 1 & 0 & 0 & 1 & 0 & 5 \\
\hline $\begin{array}{l}\text { Yang RX } \\
\text { [53] }\end{array}$ & 1 & 1 & 1 & 1 & 2 & 0 & 1 & 0 & 7 \\
\hline
\end{tabular}

${ }^{a}$ When there was no statistical significance in the response rate between case and control groups by using a chi-squared test $(P>0.05)$, one point was awarded.

${ }^{\mathrm{b}}$ Total score was calculated by adding up the points awarded in each item.

increased risk was observed in colorectal cancer. In addition, significant associations with an increased risk for the Caucasian population, but a significantly reduced risk for the Asian population, suggesting a possible ethnic difference in the genetic background and the environment, which was the similar to that reported by Wang et al [64] and Zhong et al [65]. However, the risk of different cancer types and multiethnic should be confirmed by more studies.

Although meta-analysis is robust, our study still has some limitations. Firstly, we pooled the data based on unadjusted information and lack the consideration of combination genetic factors together with environmental exposures, while a more precise analysis needs to be conducted if individual data are available. Secondly, although all eligible studies were summarized, the relatively small sample size of studies may lead to reduced statistical power when stratified according to the cancer type or ethnicity. Thirdly, the different genotyping strategies may contribute to the bias in the analysis. Fourthly, Publication bias may exist, because only published studies were included in this meta-analysis, although the result for publication bias was not statistically significant. Finally, the data sets without excluding the studies with inefficient scores base on NOS.
In summary, current data suggest that the rs 2292832 polymorphism may contribute to increased susceptibility to breast cancer, and the rs895819 polymorphism was a protective factor for cancer development among Caucasian and may contribute to breast and colorectal cancer susceptibility. Further multi-centric studies are still needed to confirm the present results.

\section{MATERIALS AND METHODS}

\section{Identification of eligible studies}

A comprehensive literature search was conducted using the PubMed, Springer, Elsevier, CNKI (Chinese), and Wanfang (Chinese) Digital Dissertations Databases for relevant articles published in English or Chinese up to July 2015 with key words 'microRNA/miR-149/miR-27a', 'rs2292832/rs895819', 'polymorphism', and 'cancer'. The full text of the candidate articles were examined carefully to determine whether they accorded with the inclusion criteria for the meta-analysis. The present study was conducted in accordance with PRISMA guidelines [66].

The inclusion criteria were as follows: 1) about the rs2292832/rs895819 polymorphisms and cancer risk, 2) based on case-control studies (including cohort studies), 3)
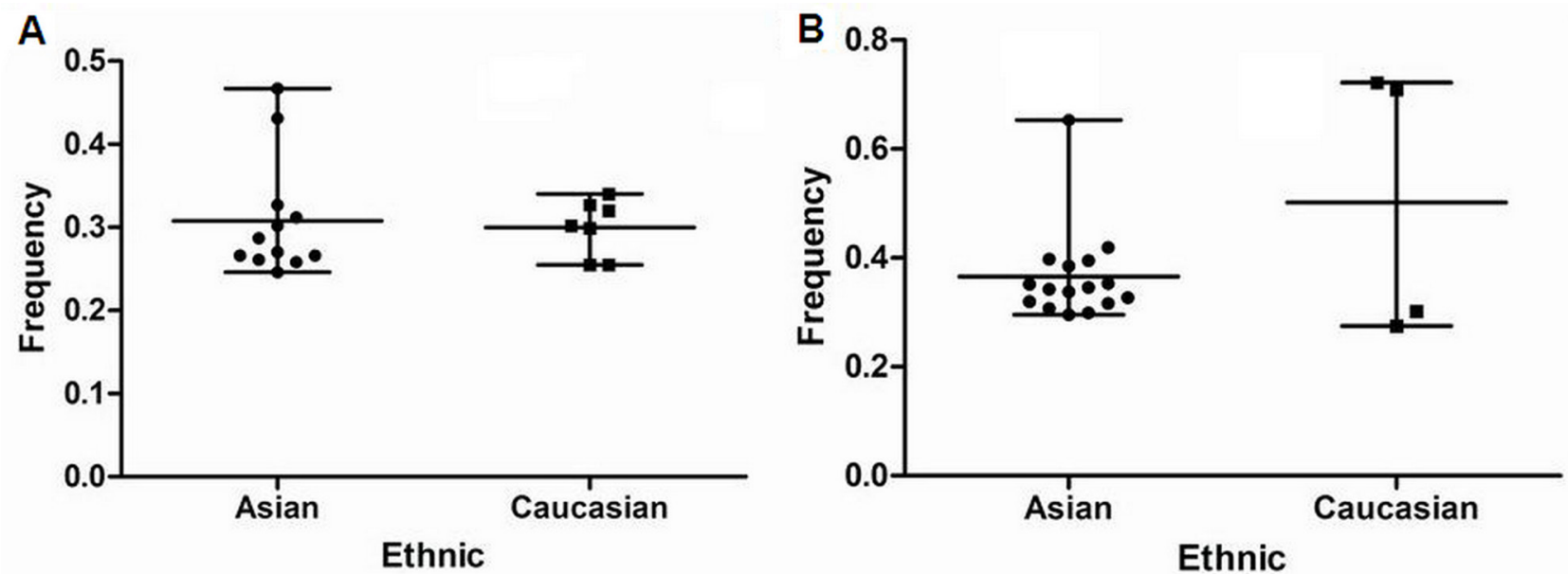

Figure 2: (A) frequencies of $\mathrm{C}$ allele in rs2292832 among controls stratified by ethnicity (B) frequencies of $\mathrm{G}$ allele in rs895819 among controls stratified by ethnicity. 
Table 3: Main results of pooled ORs of the rs2292832 and rs895819 polymorphisms on cancer risk in the meta-analysis

\begin{tabular}{|c|c|c|c|c|c|c|c|c|c|}
\hline \multirow{2}{*}{ comparisons } & \multirow{2}{*}{$\frac{\text { Cases }}{n / \mathbf{N}}$} & \multirow{2}{*}{$\frac{\text { Controls }}{\mathrm{n} / \mathbf{N}}$} & \multicolumn{3}{|c|}{ Heterogeneity test } & \multirow{2}{*}{$\begin{array}{c}\text { Summary OR } \\
(95 \% C I)\end{array}$} & \multicolumn{2}{|c|}{ Hypothesis test } & \multirow{2}{*}{ Studies } \\
\hline & & & $Q$ & $\boldsymbol{P}$ & $I^{2}(\%)$ & & $Z$ & $\boldsymbol{P}$ & \\
\hline \multicolumn{10}{|l|}{ rs2292832 } \\
\hline C vs T & $7995 / 19596$ & $8591 / 20464$ & 20.34 & 0.09 & 36 & $0.93(0.84,1.06)$ & 0.52 & 0.13 & 20 \\
\hline CT vs TT & $4129 / 7759$ & $4611 / 8511$ & 23.96 & 0.20 & 21 & $0.95(0.89,1.01)$ & 1.58 & 0.11 & 20 \\
\hline CC vs TT & $1910 / 5536$ & $2020 / 5820$ & 21.82 & 0.06 & 40 & $0.97(0.82,1.14)$ & 0.40 & 0.69 & 20 \\
\hline $\mathrm{CT}+\mathrm{CC}$ vs TT & $6039 / 9669$ & $6650 / 10550$ & 32.71 & 0.01 & 44 & $0.93(0.85,1.01)$ & 0.68 & 0.09 & 20 \\
\hline $\mathrm{CC}$ vs $\mathrm{CT}+\mathrm{TT}$ & 2068/9994 & $2182 / 10757$ & 47.55 & $<0.01$ & 51 & $1.00(0.88,1.14)$ & 0.08 & 0.94 & 21 \\
\hline \multicolumn{10}{|l|}{ rs895819 } \\
\hline G vs A & $4725 / 15804$ & $5412 / 17610$ & 43.16 & $<0.01$ & 58 & $0.99(0.91,1.17)$ & 0.09 & 0.93 & 19 \\
\hline AG vs AA & $3179 / 7062$ & $3692 / 7976$ & 30.95 & 0.03 & 45 & $0.99(0.88,1.12)$ & 0.19 & 0.85 & 19 \\
\hline GG vs AA & $798 / 4681$ & $873 / 5217$ & 27.45 & 0.04 & 42 & $1.07(0.91,1.26)$ & 0.80 & 0.42 & 19 \\
\hline $\mathrm{AG}+\mathrm{GG}$ vs AA & 3987800 & $4464 / 9060$ & 42.79 & $<0.01$ & 77 & $1.13(0.97,1.31)$ & 1.55 & 0.12 & 19 \\
\hline $\mathrm{GG}$ vs $\mathrm{AG}+\mathrm{AA}$ & $798 / 7770$ & $873 / 8911$ & 37.20 & 0.01 & 52 & $1.06(0.90,1.25)$ & 0.69 & 0.49 & 19 \\
\hline
\end{tabular}

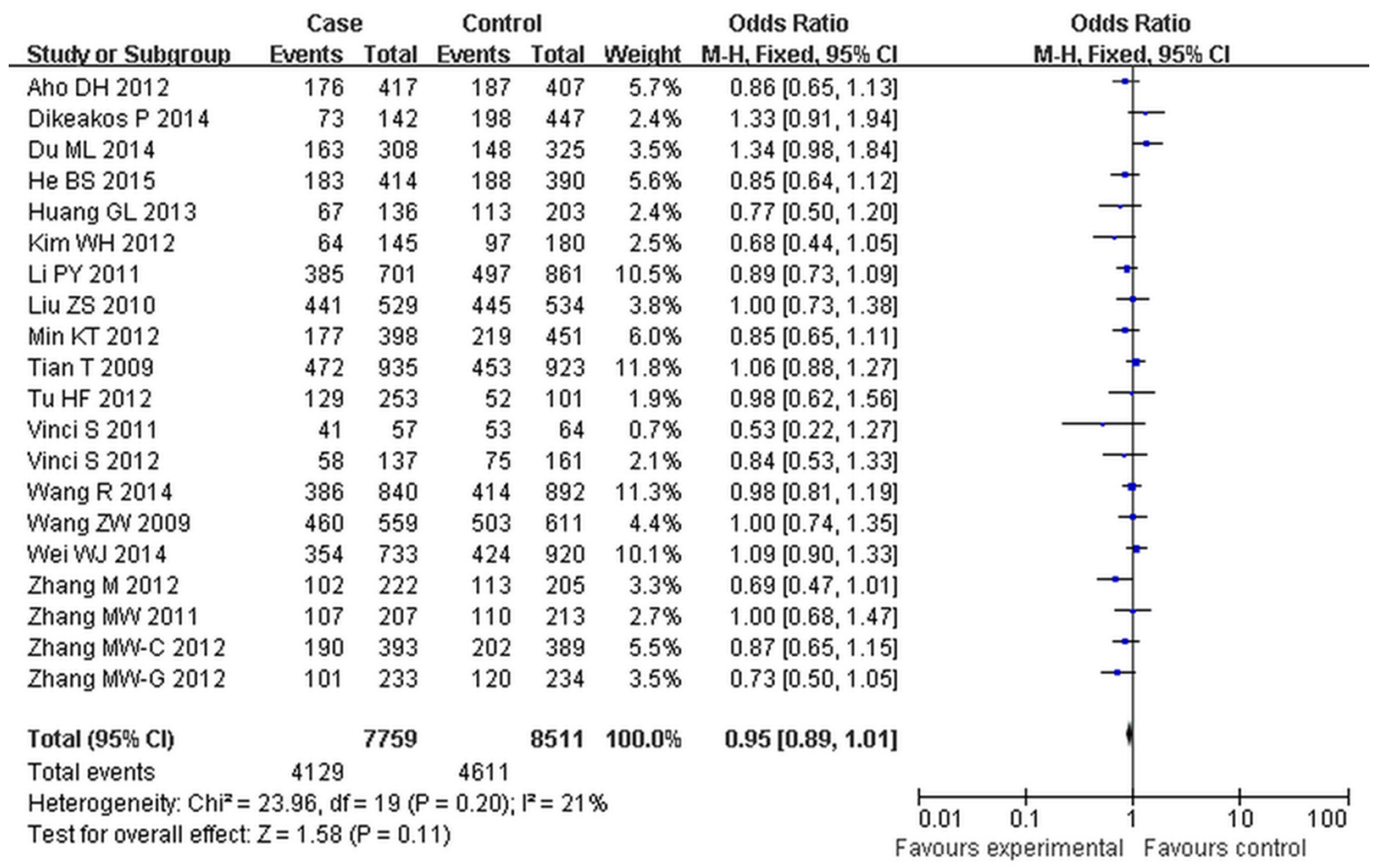

Figure 3: Forest plot of cancer risk associated with $\mathbf{r s 2 2 9 2 8 3 2}$ for the recessive model (CT vs TT). The squares and horizontal lines correspond to the study-specific OR and 95\% CI. The area of the squares reflects the study specific weight. The diamond represents the pooled OR and $95 \% \mathrm{CI}$. 
Table 4: Stratified analyses of rs2292832 polymorphism on cancer risk

\begin{tabular}{|c|c|c|c|c|c|c|c|}
\hline \multirow{2}{*}{ Comparisons } & \multicolumn{3}{|c|}{ Heterogeneity test } & \multirow{2}{*}{$\begin{array}{c}\text { Summary OR } \\
(95 \% C I)\end{array}$} & \multicolumn{2}{|c|}{ Hypothesis test } & \multirow{2}{*}{ Studies } \\
\hline & $Q$ & $P$ & $I^{2}(\%)$ & & $Z$ & $P$ & \\
\hline \multicolumn{8}{|l|}{$\begin{array}{l}\text { Ethnic } \\
\text { Asian }\end{array}$} \\
\hline $\mathrm{C}$ vs $\mathrm{T}$ & 51.04 & $<0.01$ & 49 & $0.90(0.81,1.01)$ & 1.86 & 0.06 & 16 \\
\hline CT vs TT & 18.78 & 0.22 & 20 & $0.94(0.88,1.01)$ & 1.70 & 0.09 & 16 \\
\hline $\mathrm{CC}$ vs TT & 33.84 & 0.01 & 41 & $0.93(0.78,1.11)$ & 0.79 & 0.43 & 16 \\
\hline $\mathrm{CT}+\mathrm{CC}$ vs TT & 3.93 & 0.02 & 44 & $0.94(0.87,1.03)$ & 1.31 & 0.19 & 16 \\
\hline $\mathrm{CC}$ vs $\mathrm{CT}+\mathrm{TT}$ & 32.41 & 0.02 & 38 & $1.00(0.88,1.14)$ & 0.08 & 0.94 & 16 \\
\hline \multicolumn{8}{|l|}{ Caucasian } \\
\hline C vs T & 2.55 & 0.28 & 22 & $1.06(0.84,1.33)$ & 0.47 & 0.63 & 4 \\
\hline CT vs TT & 4.73 & 0.19 & 37 & $1.02(0.82,1.25)$ & 0.14 & 0.89 & 4 \\
\hline CC vs TT & 10.45 & 0.02 & 61 & $1.16(0.67,2.01)$ & 0.54 & 0.59 & 4 \\
\hline $\mathrm{CT}+\mathrm{CC}$ vs TT & 6.09 & 0.11 & 11 & $1.08(0.88,1.31)$ & 0.72 & 0.47 & 4 \\
\hline $\mathrm{CC}$ vs $\mathrm{CT}+\mathrm{TT}$ & 8.12 & 0.09 & 51 & $1.10(0.86,1.41)$ & 0.79 & 0.43 & 5 \\
\hline \multicolumn{8}{|l|}{ Cancer types } \\
\hline \multicolumn{8}{|l|}{ Colorectal Cancer } \\
\hline C vs T & 0.79 & 0.67 & 0 & $0.97(0.85,1.10)$ & 0.48 & 0.63 & 3 \\
\hline CT vs TT & 0.02 & 0.99 & 0 & $0.85(0.71,1.02)$ & 1.72 & 0.09 & 3 \\
\hline CC vs TT & 1.02 & 0.60 & 0 & $0.94(0.71,1.25)$ & 0.42 & 0.68 & 3 \\
\hline $\mathrm{CT}+\mathrm{CC}$ vs TT & 1.12 & 0.57 & 0 & $0.87(0.67,1.15)$ & 0.97 & 0.33 & 3 \\
\hline $\mathrm{CC}$ vs $\mathrm{CT}+\mathrm{TT}$ & 0.32 & 0.96 & 0 & $1.13(0.97,1.33)$ & 1.56 & 0.12 & 3 \\
\hline \multicolumn{8}{|l|}{ Lung Cancer } \\
\hline C vs T & 3.65 & 0.16 & 45 & $0.97(0.86,1.08)$ & 0.63 & 0.53 & 3 \\
\hline CT vs TT & 1.99 & 0.37 & 0 & $0.86(0.67,1.11)$ & 1.14 & 0.25 & 3 \\
\hline CC vs TT & 4.43 & 0.11 & 55 & $0.93(0.73,1.20)$ & 0.53 & 0.60 & 3 \\
\hline $\mathrm{CT}+\mathrm{CC}$ vs TT & 1.62 & 0.44 & 0 & $1.03(0.83,1.28)$ & 0.25 & 0.80 & 3 \\
\hline $\mathrm{CC}$ vs $\mathrm{CT}+\mathrm{TT}$ & 3.28 & 0.19 & 39 & $0.96(0.83,1.12)$ & 0.48 & 0.63 & 3 \\
\hline \multicolumn{8}{|l|}{ Breast Cancer } \\
\hline $\mathrm{C}$ vs T & 13.72 & $<0.01$ & 55 & $0.82(0.61,1.10)$ & 1.31 & 0.19 & 3 \\
\hline CT vs TT & 2.19 & 0.33 & 9 & $0.86(0.72,1.03)$ & 1.64 & 0.10 & 3 \\
\hline CC vs TT & 5.81 & 0.55 & 46 & $0.82(0.65,1.03)$ & 1.73 & 0.08 & 3 \\
\hline $\mathrm{CT}+\mathrm{CC}$ vs TT & 2.72 & 0.26 & 26 & $0.83(0.70,0.98)$ & 2.18 & 0.03 & 3 \\
\hline $\mathrm{CC}$ vs $\mathrm{CT}+\mathrm{TT}$ & 2.82 & 0.24 & 29 & $0.80(0.68,0.93)$ & 2.81 & 0.00 & 3 \\
\hline \multicolumn{8}{|l|}{ Other cancers } \\
\hline C vs T & 13.42 & 0.06 & 45 & $0.91(0.78,1.05)$ & 1.29 & 0.20 & 11 \\
\hline CT vs TT & 19.35 & 0.04 & 48 & $0.96(0.85,1.08)$ & 0.75 & 0.45 & 11 \\
\hline $\mathrm{CC}$ vs TT & 16.28 & 0.02 & 57 & $1.06(0.83,1.35)$ & 0.47 & 0.64 & 11 \\
\hline $\mathrm{CT}+\mathrm{CC}$ vs TT & 13.67 & 0.09 & 41 & $1.06(0.96,1.16)$ & 1.17 & 0.24 & 11 \\
\hline $\mathrm{CC}$ vs $\mathrm{CT}+\mathrm{TT}$ & 5.98 & 0.54 & 0 & $1.18(1.06,1.31)$ & 3.14 & 0.00 & 12 \\
\hline \multicolumn{8}{|l|}{$\begin{array}{l}\text { Source of control } \\
\text { Population }\end{array}$} \\
\hline C vs T & 78.91 & $<0.01$ & 60 & $0.92(0.83,1.02)$ & 1.53 & 0.13 & 17 \\
\hline CT vs TT & 20.50 & 0.20 & 22 & $0.95(0.88,1.01)$ & 1.59 & 0.11 & 17 \\
\hline
\end{tabular}




\begin{tabular}{|l|c|c|c|c|c|c|c|}
\hline CC vs TT & 29.47 & 0.02 & 46 & $1.00(0.86,1.16)$ & 0.04 & 0.97 & 17 \\
\hline CT + CC vs TT & 26.00 & 0.05 & 38 & $0.96(0.90,1.03)$ & 1.06 & 0.29 & 17 \\
\hline CC vs CT + TT & 27.06 & 0.06 & 38 & $1.01(0.94,1.10)$ & 0.32 & 0.75 & 18 \\
\hline Hospital & & & & & & & \\
\hline C vs T & 13.71 & 0.01 & 65 & $0.97(0.68,1.38)$ & 0.17 & 0.86 & 3 \\
\hline CT vs TT & 3.34 & 0.19 & 40 & $0.98(0.83,1.15)$ & 0.30 & 0.77 & 3 \\
\hline CC vs TT & 17.29 & $<0.01$ & 68 & $0.83(0.64,2.03)$ & 0.40 & 0.69 & 3 \\
\hline CT + CC vs TT & 7.75 & 0.02 & 64 & $0.99(0.69,1.43)$ & 0.05 & 0.96 & 3 \\
\hline CC vs CT + TT & 15.24 & $<0.01$ & 67 & $0.82(0.57,1.80)$ & 0.49 & 0.62 & 3 \\
\hline Sample size & & & & & & & \\
\hline$\geq 300$ & & & & & & & \\
\hline C vs T & 76.76 & $<0.01$ & 66 & $0.99(0.87,1.12)$ & 0.19 & 0.85 & 12 \\
\hline CT vs TT & 12.83 & 0.30 & 14 & $0.99(0.92,1.06)$ & 0.34 & 0.74 & 12 \\
\hline CC vs TT & 35.37 & $<0.01$ & 59 & $1.04(0.86,1.26)$ & 0.42 & 0.68 & 12 \\
\hline CT + CC vs TT & 21.90 & 0.03 & 50 & $1.00(0.91,1.10)$ & 0.04 & 0.97 & 12 \\
\hline CC vs CT + TT & 30.33 & $<0.01$ & 64 & $1.03(0.90,1.19)$ & 0.47 & 0.64 & 13 \\
\hline$<300$ & & & & & & & \\
\hline C vs T & 7.50 & 0.38 & 7 & $0.92(0.94,1.11)$ & 1.88 & 0.06 & 8 \\
\hline CT vs TT & 4.34 & 0.74 & 0 & $0.89(0.78,1.02)$ & 1.74 & 0.08 & 8 \\
\hline CC vs TT & 12.99 & 0.07 & 46 & $0.82(0.65,1.04)$ & 1.66 & 0.10 & 8 \\
\hline CT + CC vs TT & 5.03 & 0.66 & 0 & $0.90(0.80,1.03)$ & 1.70 & 0.09 & 8 \\
\hline CC vs CT + TT & 13.13 & 0.07 & 47 & $0.93(0.75,1.14)$ & 0.73 & 0.47 & 8 \\
\hline
\end{tabular}

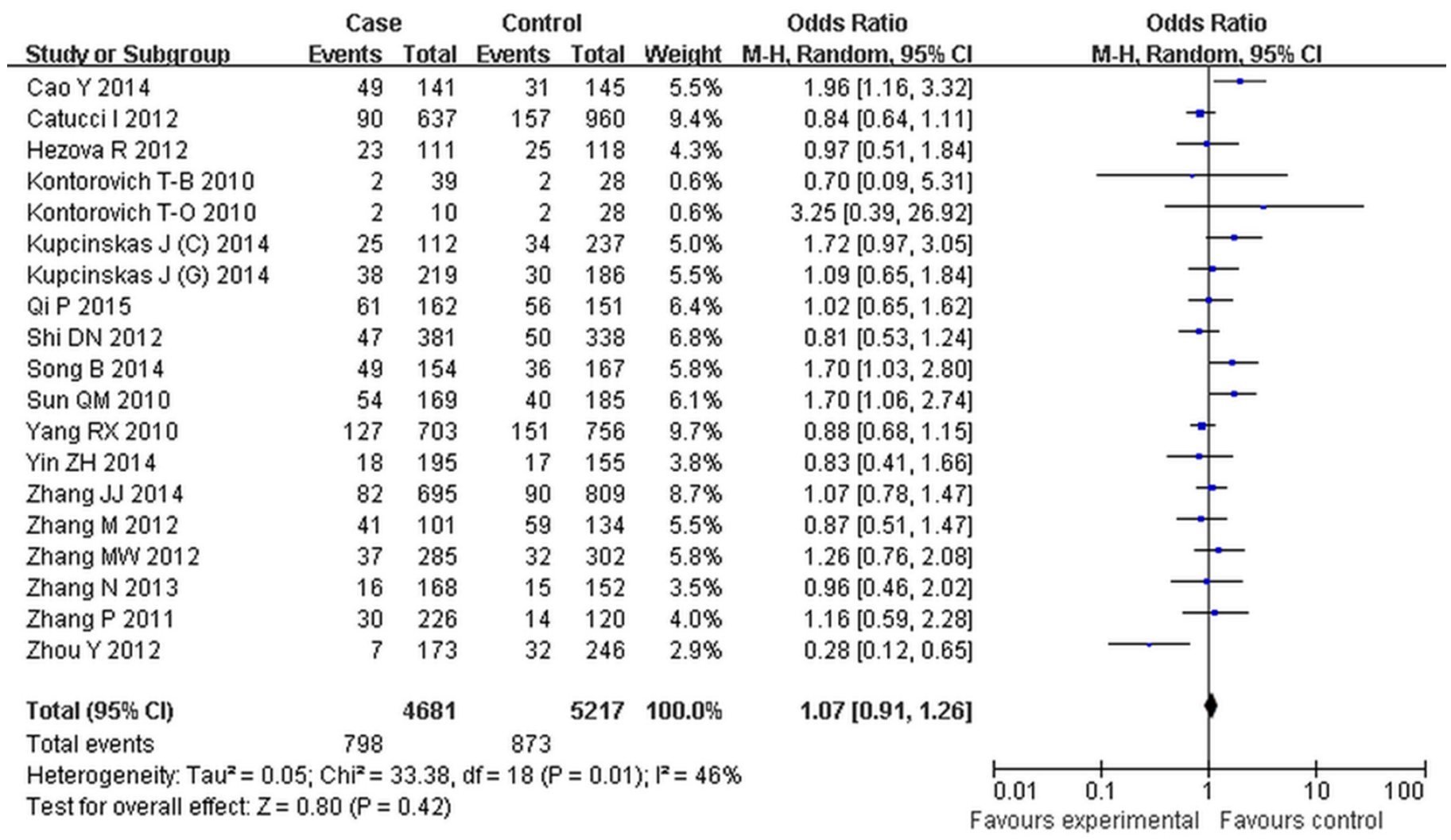

Figure 4: Forest plot of cancer risk associated with rs895819 for the GG vs AA compared with the AA genotype. 
Table 5: Stratified analyses of the rs895819 polymorphism on cancer risk

\begin{tabular}{|c|c|c|c|c|c|c|c|}
\hline \multirow{2}{*}{ Comparisons } & \multicolumn{3}{|c|}{ Heterogeneity test } & \multirow{2}{*}{$\begin{array}{c}\text { Summary OR } \\
(95 \% C I)\end{array}$} & \multicolumn{2}{|c|}{ Hypothesis test } & \multirow{2}{*}{ Studies } \\
\hline & $Q$ & $P$ & $I^{2}(\%)$ & & $Z$ & $P$ & \\
\hline \multicolumn{8}{|l|}{ Ethnic } \\
\hline \multicolumn{8}{|l|}{ Asian } \\
\hline G vs A & 34.11 & $<0.01$ & 68 & $1.02(0.91,1.14)$ & 0.27 & 0.79 & 12 \\
\hline AG vs AA & 27.19 & 0.01 & 60 & $1.09(0.95,1.26)$ & 1.25 & 0.21 & 12 \\
\hline GG vs AA & 24.68 & 0.01 & 55 & $1.09(0.87,1.37)$ & 0.73 & 0.47 & 12 \\
\hline $\mathrm{AG}+\mathrm{GG}$ vs AA & 53.69 & $<0.01$ & 80 & $1.24(1.03,1.50)$ & 2.28 & 0.02 & 12 \\
\hline $\mathrm{GG}$ vs $\mathrm{AG}+\mathrm{AA}$ & 30.73 & $<0.01$ & 64 & $1.03(0.81,1.31)$ & 0.25 & 0.80 & 12 \\
\hline \multicolumn{8}{|l|}{ Caucasian } \\
\hline G vs A & 6.91 & 0.33 & 13 & $0.92(0.86,0.99)$ & 2.27 & 0.02 & 7 \\
\hline AG vs AA & 7.70 & 0.26 & 22 & $0.81(0.73,0.89)$ & 3.82 & 0.00 & 7 \\
\hline GG vs AA & 6.74 & 0.35 & 11 & $0.95(0.80,1.12)$ & 0.65 & 0.51 & 7 \\
\hline $\mathrm{AG}+\mathrm{GG}$ vs AA & 4.17 & 0.65 & 0 & $0.87(0.79,0.95)$ & 2.69 & 0.00 & 7 \\
\hline $\mathrm{GG}$ vs $\mathrm{AG}+\mathrm{AA}$ & 6.47 & 0.37 & 7 & $1.03(0.88,1.02)$ & 0.34 & 0.74 & 7 \\
\hline \multicolumn{8}{|l|}{ Breast cancer } \\
\hline G vs A & 8.76 & 0.12 & 43 & $0.92(0.86,0.99)$ & 2.15 & 0.03 & 6 \\
\hline AG vs AA & 11.41 & 0.04 & 56 & $0.83(0.75,0.92)$ & 3.51 & 0.00 & 6 \\
\hline GG vs AA & 1.17 & 0.95 & 0 & $0.90(0.76,1.07)$ & 1.21 & 0.23 & 6 \\
\hline $\mathrm{AG}+\mathrm{GG}$ vs AA & 5.80 & 0.33 & 14 & $0.88(0.80,0.97)$ & 2.58 & 0.01 & 6 \\
\hline $\mathrm{GG}$ vs $\mathrm{AG}+\mathrm{AA}$ & 2.40 & 0.79 & 0 & $0.98(0.84,1.15)$ & 0.24 & 0.81 & 6 \\
\hline \multicolumn{8}{|l|}{ Gastric cancer } \\
\hline G vs $A$ & 16.96 & 0.00 & 62 & $1.11(0.84,1.46)$ & 0.70 & 0.48 & 4 \\
\hline AG vs AA & 10.15 & 0.02 & 50 & $1.08(0.80,1.47)$ & 0.50 & 0.42 & 4 \\
\hline GG vs AA & 15.44 & 0.00 & 60 & $1.05(0.55,1.99)$ & 0.15 & 0.88 & 4 \\
\hline $\mathrm{AG}+\mathrm{GG}$ vs $\mathrm{AA}$ & 13.52 & 0.00 & 58 & $1.10(0.79,1.53)$ & 0.55 & 0.58 & 4 \\
\hline $\mathrm{GG}$ vs $\mathrm{AG}+\mathrm{AA}$ & 12.52 & 0.01 & 56 & $1.02(0.59,1.76)$ & 0.07 & 0.94 & 4 \\
\hline \multicolumn{8}{|l|}{ Colorectal Cancer } \\
\hline G vs $\mathrm{A}$ & 1.78 & 0.62 & 0 & $1.07(0.94,1.21)$ & 1.06 & 0.29 & 4 \\
\hline $\mathrm{AG}$ vs AA & 3.42 & 0.33 & 12 & $1.14(0.96,1.35)$ & 1.47 & 0.14 & 4 \\
\hline GG vs AA & 3.40 & 0.33 & 12 & $1.45(1.10,1.92)$ & 2.66 & 0.00 & 4 \\
\hline $\mathrm{AG}+\mathrm{GG}$ vs $\mathrm{AA}$ & 7.81 & 0.05 & 62 & $1.35(1.15,1.58)$ & 3.65 & 0.00 & 4 \\
\hline $\mathrm{GG}$ vs $\mathrm{AG}+\mathrm{AA}$ & 2.52 & 0.47 & 0 & $1.36(1.04,1.77)$ & 2.27 & 0.02 & 4 \\
\hline \multicolumn{8}{|l|}{ Other cancers } \\
\hline G vs $\mathrm{A}$ & 2.12 & 0.55 & 0 & $0.87(0.79,0.96)$ & 2.87 & 0.00 & 4 \\
\hline AG vs AA & 7.08 & 0.07 & 58 & $0.92(0.81,1.04)$ & 1.30 & 0.19 & 4 \\
\hline GG vs AA & 2.49 & 0.48 & 0 & $0.96(0.76,1.22)$ & 0.30 & 0.77 & 4 \\
\hline $\mathrm{AG}+\mathrm{GG}$ vs AA & 22.87 & 0.00 & 70 & $1.26(0.77,2.07)$ & 0.92 & 0.36 & 4 \\
\hline $\mathrm{GG}$ vs $\mathrm{AG}+\mathrm{AA}$ & 1.70 & 0.64 & 0 & $1.05(0.84,1.33)$ & 0.45 & 0.65 & 4 \\
\hline \multicolumn{8}{|l|}{$\begin{array}{l}\text { Source of control } \\
\text { Population }\end{array}$} \\
\hline G vs $\mathrm{A}$ & 28.89 & 0.01 & 58 & $0.99(0.90,1.10)$ & 0.18 & 0.86 & 13 \\
\hline AG vs AA & 43.20 & 0.00 & 72 & $1.02(0.86,1.21)$ & 0.22 & 0.83 & 13 \\
\hline GG vs AA & 14.44 & 0.27 & 17 & $1.06(0.93,1.21)$ & 0.83 & 0.41 & 13 \\
\hline $\mathrm{AG}+\mathrm{GG}$ vs AA & 61.57 & 0.00 & 81 & $1.14(0.94,1.38)$ & 1.36 & 0.17 & 13 \\
\hline $\mathrm{GG}$ vs $\mathrm{AG}+\mathrm{AA}$ & 20.53 & 0.06 & 42 & $1.03(0.91,1.17)$ & 0.46 & 0.65 & 13 \\
\hline \multicolumn{8}{|l|}{ Hospital } \\
\hline G vs $A$ & 14.18 & 0.01 & 65 & $0.99(0.86,1.15)$ & 0.08 & 0.94 & 6 \\
\hline $\mathrm{AG}$ vs AA & 7.78 & 0.17 & 36 & $0.94(0.84,1.05)$ & 1.11 & 0.27 & 6 \\
\hline GG vs AA & 18.75 & 0.00 & 73 & $0.98(0.65,1.49)$ & 0.08 & 0.94 & 6 \\
\hline $\mathrm{AG}+\mathrm{GG}$ vs $\mathrm{AA}$ & 27.21 & 0.00 & 82 & $1.10(0.84,1.43)$ & 0.68 & 0.50 & 6 \\
\hline
\end{tabular}




\begin{tabular}{|l|c|c|c|c|c|c|c|}
\hline GG vs AG + AA & 16.68 & 0.01 & 70 & $1.06(0.73,1.55)$ & 0.32 & 0.75 & 6 \\
\hline Sample size & & & & & & & \\
\hline$\geq 300$ & & & & & & & \\
\hline G vs A & 22.21 & 0.02 & 59 & $0.95(0.87,1.04)$ & 1.16 & 0.25 & 10 \\
\hline AG vs AA & 27.95 & 0.01 & 68 & $0.92(0.80,1.05)$ & 1.23 & 0.22 & 10 \\
\hline GG vs AA & 21.34 & 0.01 & 58 & $0.99(0.80,1.23)$ & 0.05 & 0.96 & 10 \\
\hline AG + GG vs AA & 76.99 & 0.00 & 88 & $1.09(0.88,1.35)$ & 0.77 & 0.44 & 10 \\
\hline GG vs AG + AA & 17.22 & 0.05 & 48 & $1.03(0.91,1.16)$ & 0.42 & 0.67 & 10 \\
\hline$<300$ & & & & & & & \\
\hline G vs A & 13.95 & 0.08 & 43 & $1.08(0.98,1.18)$ & 1.45 & 0.15 & 9 \\
\hline AG vs AA & 12.81 & 0.12 & 38 & $1.15(1.00,1.33)$ & 2.02 & 0.04 & 9 \\
\hline GG vs AA & 8.96 & 0.35 & 11 & $1.22(0.99,1.50)$ & 1.85 & 0.06 & 9 \\
\hline AG + GG vs AA & 9.82 & 0.28 & 19 & $1.19(0.98,1.32)$ & 1.74 & 0.07 & 9 \\
\hline GG vs AG + AA & 19.99 & 0.01 & 60 & $1.08(0.77,1.50)$ & 0.44 & 0.66 & 9 \\
\hline
\end{tabular}

Table 6: The results of heterogeneity test for $\mathbf{r s 2 2 9 2 8 3 2}$ and $\mathbf{r s 8 9 5 8 1 9}$

Comparisons
\begin{tabular}{|l|c|c|c|c|c|c|c|c|c|c|}
\hline $\begin{array}{c}\text { Publication } \\
\text { year }\end{array}$ & Ethnicity & \multicolumn{2}{c}{$\begin{array}{c}\text { Cancer } \\
\text { type }\end{array}$} & \multicolumn{2}{c}{ Match } & \multicolumn{2}{c}{ Language $\begin{array}{c}\text { Source of } \\
\text { control }\end{array}$} & \multicolumn{2}{c}{$\begin{array}{c}\text { Assay } \\
\text { size }\end{array}$} & $\begin{array}{c}\text { Quality } \\
\text { control }\end{array}$ \\
\hline rs2292832 & & & & & & & & & \\
\hline C vs T & 0.737 & 0.339 & 0.256 & 0.812 & 0.653 & 0.547 & 0.417 & 0.291 & 0.781 \\
\hline CT vs TT & 0.392 & 0.440 & 0.331 & 0.329 & 0.220 & 0.514 & 0.519 & 0.765 & 0.529 \\
\hline CC vs TT & 0.388 & 0.838 & 0.463 & 0.784 & 0.463 & 0.875 & 0.772 & 0.573 & 0.514 \\
\hline CT + CC vs TT & 0.737 & 0.440 & 0.547 & 0.956 & 0.853 & 0.443 & 0.949 & 0.552 & 0.554 \\
\hline CC vs CT + TT & 0.519 & 0.519 & 0.440 & 0.331 & 0.389 & 0.396 & 0.838 & 0.336 & 0.815 \\
\hline rs895819 & & & & & & & & & \\
\hline G vs A & 0.418 & 0.426 & 0.275 & 0.581 & 0.593 & 0.581 & 0.336 & 0.581 & 0.225 \\
\hline AG vs AA & 0.440 & 0.841 & 0.415 & 0.797 & 0.596 & 0.797 & 0.554 & 0.797 & 0.442 \\
\hline GG vs AA & 0.838 & 0.721 & 0.487 & 0.998 & 0.827 & 0.498 & 0.423 & 0.998 & 0.366 \\
\hline AG + GG vs AA & 0.418 & 0.426 & 0.159 & 0.989 & 0.656 & 0.989 & 0.359 & 0.989 & 0.396 \\
\hline GG vs AG + AA & 0.327 & 0.841 & 0.881 & 0.077 & 0.914 & 0.077 & 0.073 & 0.077 & 0.990 \\
\hline
\end{tabular}

Table 7: Publication bias of rs2292832 and rs895819 for Egger's test

\begin{tabular}{|l|c|c|c|}
\hline \multicolumn{1}{c|}{$\boldsymbol{t}$} & $\boldsymbol{p}$ & $\mathbf{9 5 \%} \boldsymbol{C I}$ \\
\hline rs2292832 & & & $-1.657 \sim 4.245$ \\
\hline T vs C & 0.96 & 0.358 & $-1.748 \sim 1.151$ \\
\hline CT vs CC & -0.45 & 0.661 & $-1.171 \sim 3.001$ \\
\hline TT vs CC & 0.96 & 0.358 & $-1.256 \sim 1.777$ \\
\hline CT + TT vs CC & 0.37 & 0.715 & $-0.572 \sim 3.100$ \\
\hline TT vs CT + CC & 1.60 & 0.083 & \\
\hline rs895819 & & & $-2.337 \sim 3.452$ \\
\hline G vs A & 0.44 & 0.673 & $-1.122 \sim 3.555$ \\
\hline AG vs AA & 1.18 & 0.270 & $-1.792 \sim 2.291$ \\
\hline GG vs AA & 0.28 & 0.789 & $-1.219 \sim 3.612$ \\
\hline AG + GG vs AA & 1.12 & 0.292 & $-1.923 \sim 1.803$ \\
\hline GG vs AG + AA & -0.07 & 0.943 & \\
\hline
\end{tabular}


Begg's funnel plot with pseudo $95 \%$ confidence limits

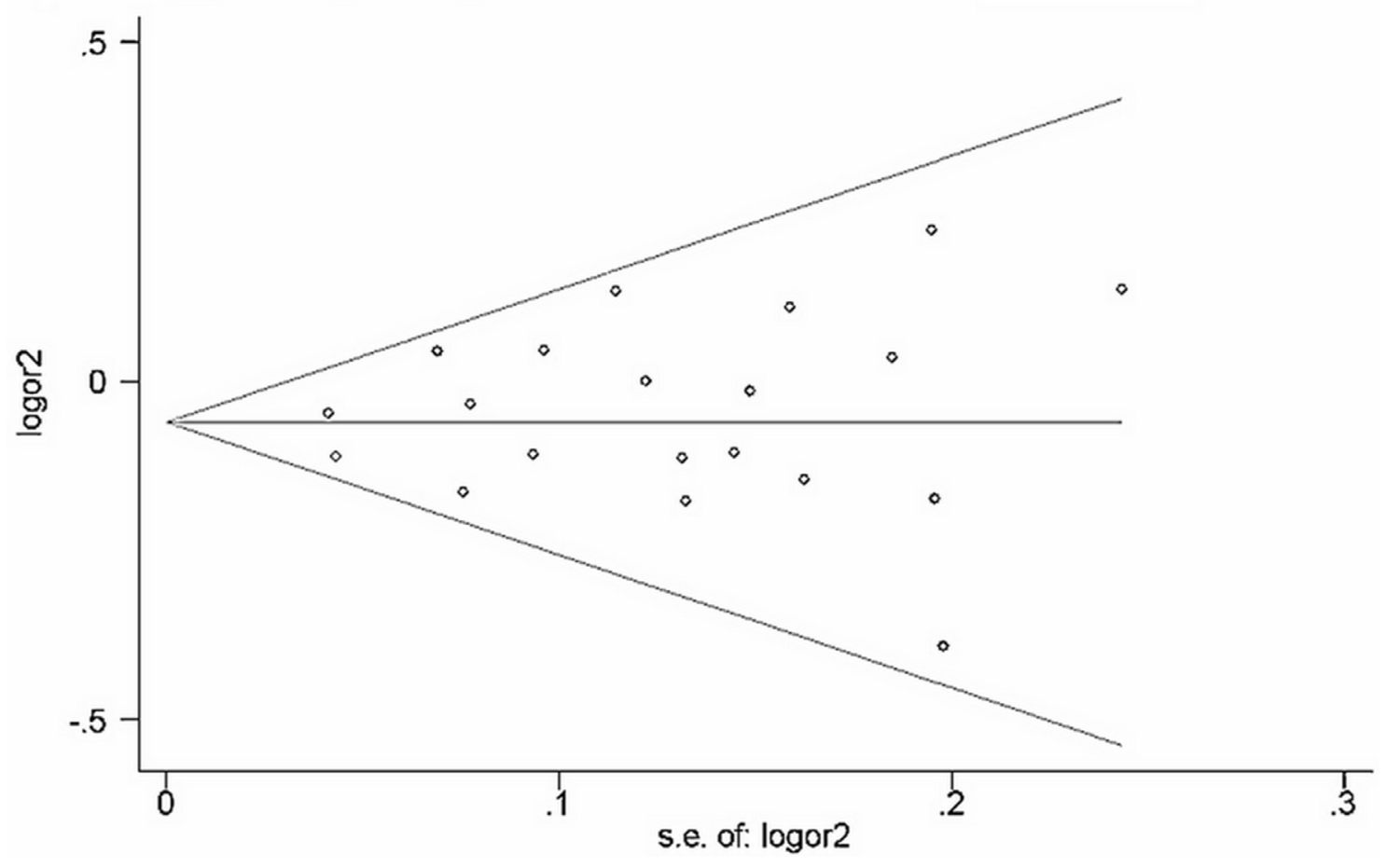

Figure 5: Funnel plot of rs2292832 polymorphism and cancer risk for dominant models (TT + CT vs CC). The horizontal line in the funnel plot indicates the fixed-effects summary estimate, whereas the sloping lines indicate the expected $95 \% \mathrm{CI}$ for a given SE.

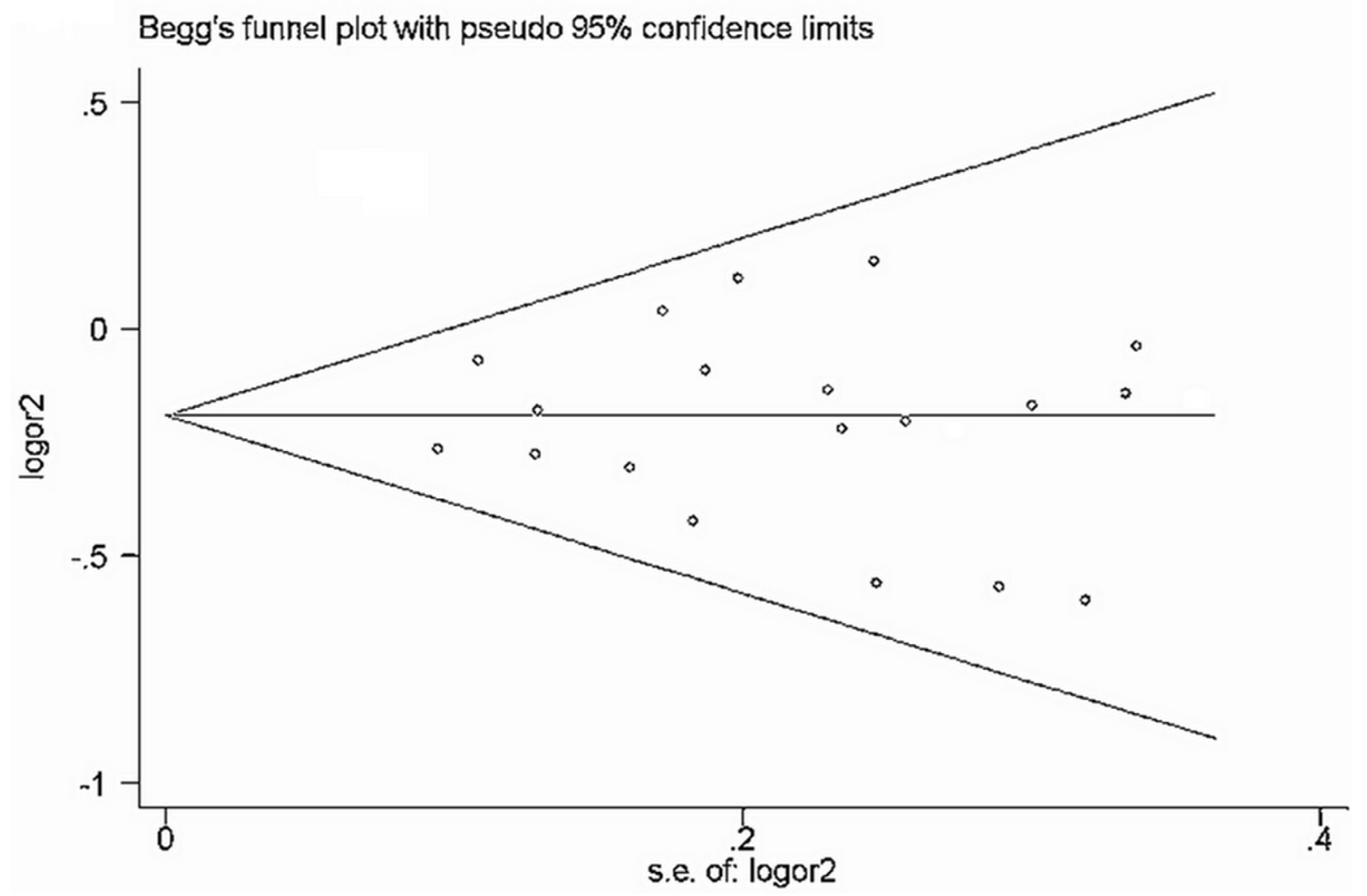

Figure 6: Funnel plot of rs895819 polymorphism and cancer risk for dominant models (TT + CT vs CC). 
sufficient published data for estimating an odds ratio (OR) with 95\% confidence interval (CI), and 4) genotype distribution of control groups must be in accordance with the assumptions of Hardy-Weinberg equilibrium (HWE).

In case of redundant publications, only the studies with the largest sample size and/or latest published date were included.

\section{Data extraction}

Data were extracted independently by two investigators (YJF and FJD). Data for analyses, including first author, publication year, cancer type, country of origin, ethnicity, study design, genotype detection methods and quality control or not. If discrepancies existed, consensus would be finally reached on discussion.

\section{Quality assessment}

Quality assessment criteria were utilized to evaluate methodological quality of included studies based on Newcastle-Ottawa Scale (NOS) [67] for quality of case-control. A nine-point scale of the NOS (range, 0-9 points) has been developed for the evaluation, a high-quality study was defined as one with a score of $\geq 7$.

\section{Statistical analysis}

The analyses were conducted in Review Manager 5.0 (Version 5 for Windows, Cochrane Collaboration, Oxford, UK). The overall strength of an association between rs2292832 and rs895819 polymorphisms and cancer risk assessed by crude ORs together with their corresponding 95\% CIs. The stratified analysis was conducted by ethnicity (Asians, Caucasians), cancer type, source of control and sample size (300 as the boundary).

Heterogeneity in meta-analysis refers to the variation in study outcomes between different studies. Between-study heterogeneity was evaluated with a $\chi^{2}$ based $Q$-test among the studies [68]. Heterogeneity was considered significant when $P<0.05$. In case of no significant heterogeneity, point estimates and 95\% CI was estimated using the fixed effect model (Mantel-Haenszel), otherwise, random effects model (DerSimonian Laird) was employed $[69,70]$. The significance of overall OR was determined by the $Z$-test.

If there were significant heterogeneity among included studies, the sources of heterogeneity would be explored using meta-regression in Stata 12.0 (StataCorp, College Station, TX, USA). To assess the stability of the results, one-way sensitivity analyses were performed to assess the stability of the results, in which a single study in the meta-analysis was deleted each time to reflect the influence of the individual data set to the pooled OR. The publication bias was diagnosed by using inverted funnel plots, Begg's test and the Egger's test by Stata
12.0. Statistical tests performed in the present analysis were considered significant whenever the corresponding null-hypothesis probability was $P<0.05$.

\section{ACKNOWLEDGMENTS AND FUNDING}

This research was supported by National Natural Science Foundation of China (No.81202278), and Program for Science and Technology Key Projects of Henan Province (201303005).

\section{CONFLICTS OF INTEREST}

None declared.

\section{REFERENCES}

1. Ambros V. The functions of animal microRNAs. Nature. 2004; 431:350-355.

2. Krek A, Grun D, Poy MN, Wolf R, Rosenberg L, Epstein EJ, MacMenamin P, da Piedade I, Gunsalus KC, Stoffel M, Rajewsky N. Combinatorial microRNA target predictions. Nature genetics. 2005; 37:495-500.

3. Berezikov E, Guryev V, van de Belt J, Wienholds E, Plasterk RH, Cuppen E. Phylogenetic shadowing and computational identification of human microRNA genes. Cell. 2005; 120:21-24.

4. Kong YW, Ferland-McCollough D, Jackson TJ, Bushell M. microRNAs in cancer management. The lancet oncology. 2012; 13:e249-258.

5. Ryan BM, Robles AI, Harris CC. Genetic variation in microRNA networks: the implications for cancer research. Nature reviews Cancer. 2010; 10:389-402.

6. Xu W, Xu J, Liu S, Chen B, Wang X, Li Y, Qian Y, Zhao W, $\mathrm{Wu}$ J. Effects of common polymorphisms rs11614913 in miR-196a2 and rs2910164 in miR-146a on cancer susceptibility: a meta-analysis. PloS one. 2011; 6:e20471.

7. Horikawa Y, Wood CG, Yang H, Zhao H, Ye Y, Gu J, Lin J, Habuchi T, Wu X. Single nucleotide polymorphisms of microRNA machinery genes modify the risk of renal cell carcinoma. Clinical cancer research. 2008; 14:7956-7962.

8. George GP, Gangwar R, Mandal RK, Sankhwar SN, Mittal RD. Genetic variation in microRNA genes and prostate cancer risk in North Indian population. Molecular biology reports. 2011; 38:1609-1615.

9. Hu Y, Yu CY, Wang JL, Guan J, Chen HY, Fang JY. MicroRNA sequence polymorphisms and the risk of different types of cancer. Scientific reports. 2014; 4:3648.

10. Ma XP, Zhang T, Peng B, Yu L, Jiang de K. Association between microRNA polymorphisms and cancer risk based on the findings of 66 case-control studies. PloS one. 2013; 8:e79584.

11. He B, Pan Y, Xu Y, Deng Q, Sun H, Gao T, Wang S. Associations of polymorphisms in microRNAs with female breast cancer risk in Chinese population. Tumour biology. 2015. 
12. Du M, Lu D, Wang Q, Chu H, Tong N, Pan X, Qin C, Yin C, Wang M, Zhang Z. Genetic variations in microRNAs and the risk and survival of renal cell cancer. Carcinogenesis. 2014; 35:1629-1635.

13. Dikeakos P, Theodoropoulos G, Rizos S, Tzanakis N, Zografos G, Gazouli M. Association of the miR-146aC $>$ G, miR-149T $>$ C, and miR-196a2T $>$ C polymorphisms with gastric cancer risk and survival in the Greek population. Molecular biology reports. 2014; 41:1075-1080.

14. Pu JY, Dong W, Zhang L, Liang WB, Yang Y, Lv ML. No association between single nucleotide polymorphisms in pre-mirnas and the risk of gastric cancer in Chinese population. Iranian journal of basic medical sciences. 2014; 17:128-133.

15. Wei WJ, Lu ZW, Li DS, Wang Y, Zhu YX, Wang ZY, Wu Y, Wang YL, Ji QH. Association of the miR-149 Rs2292832 polymorphism with papillary thyroid cancer risk and clinicopathologic characteristics in a Chinese population. Int J Mol Sci. 2014; 15:20968-20981.

16. Wang R, Zhang J, Ma Y, Chen L, Guo S, Zhang X, Wu L, Pei X, Liu S, Wang J, Hu H, Liu J. Association study of miR149 rs2292832 and miR608 rs4919510 and the risk of hepatocellular carcinoma in a largescale population. Molecular medicine reports. 2014; 10:2736-2744.

17. Wu R. (2014). The Association of miR-SNP with the Susceptibility of Colorectal Cancer and Response to Chemotherapy. Soochow University).

18. Huang GL, Lu Y, Pu XX, He YX, Chen ML, Li YZ, Tang SY, Che H, He Z. Association study between miR-149 gene polymorphism and nasopharyngeal carcinoma. Biomed Rep. 2013; 1:599-603.

19. Chu YH, Hsieh MJ, Chiou HL, Liou YS, Yang CC, Yang SF, Kuo WH. MicroRNA gene polymorphisms and environmental factors increase patient susceptibility to hepatocellular carcinoma. PloS one. 2014; 9:e89930.

20. Lv M, Dong W, Li L, Zhang L, Su X, Wang L, Gao L. Association between genetic variants in pre-miRNA and colorectal cancer risk in a Chinese population. Journal of cancer research and clinical oncology. 2013; 139: 1405-1410.

21. Song X, Sturgis EM, Liu J, Jin L, Wang Z, Zhang C, Wei Q, Li G. MicroRNA variants increase the risk of HPV-associated squamous cell carcinoma of the oropharynx in never smokers. PloS one. 2013; 8:e56622.

22. Tu HF, Liu CJ, Chang CL, Wang PW, Kao SY, Yang CC, Yu EH, Lin SC, Chang KW. The association between genetic polymorphism and the processing efficiency of miR-149 affects the prognosis of patients with head and neck squamous cell carcinoma. PloS one. 2012; 7:e51606.

23. Zhang M, Jin M, Yu Y, Zhang S, Wu Y, Liu H, Chen B, Li Q, Ma X, Chen K. Associations of miRNA polymorphisms and female physiological characteristics with breast cancer risk in Chinese population. European journal of cancer care. 2012; 21:274-280.

24. Zhang MW, Jin MJ, Yu YX, Zhang SC, Liu B, Jiang X, Pan YF, Li QI, Ma SY, Chen K. Associations of lifestyle-related factors, hsa-miR-149 and hsa-miR-605 gene polymorphisms with gastrointestinal cancer risk. Molecular carcinogenesis. 2012; 51:E21-31.

25. Min KT, Kim JW, Jeon YJ, Jang MJ, Chong SY, Oh D, Kim NK. Association of the miR-146aC $>$ G, 149C $>$ T, $196 \mathrm{a} 2 \mathrm{C}>\mathrm{T}$, and 499A $>\mathrm{G}$ polymorphisms with colorectal cancer in the Korean population. Molecular carcinogenesis. 2012; 51:E65-73.

26. Ahn DH, Rah H, Choi YK, Jeon YJ, Min KT, Kwack K, Hong SP, Hwang SG, Kim NK. Association of the $\operatorname{miR}-146 \mathrm{aC}>\mathrm{G}, \quad \operatorname{miR}-149 \mathrm{~T}>\mathrm{C}$, miR-196a2T $>\mathrm{C}$, and miR-499A $>\mathrm{G}$ polymorphisms with gastric cancer risk and survival in the Korean population. Molecular carcinogenesis. 2012.

27. Kim WH, Min KT, Jeon YJ, Kwon CI, Ko KH, Park PW, Hong SP, Rim KS, Kwon SW, Hwang SG, Kim NK. Association study of microRNA polymorphisms with hepatocellular carcinoma in Korean population. Gene. 2012; 504:92-97.

28. Vinci S, Gelmini S, Mancini I, Malentacchi F, Pazzagli M, Beltrami C, Pinzani P, Orlando C. Genetic and epigenetic factors in regulation of microRNA in colorectal cancers. Methods. 2013; 59:138-146.

29. Vinci S, Gelmini S, Pratesi N, Conti S, Malentacchi F, Simi L, Pazzagli M, Orlando C. Genetic variants in miR-146a, miR-149, miR-196a2, miR-499 and their influence on relative expression in lung cancers. Clinical chemistry and laboratory medicine: CCLM/FESCC. 2011; 49:2073-2080.

30. Li PY. (2011). Genetic associations of miRNA-SNPs with the common diseases and integrative analysis of "OMICS" data of hepatocellular carcinoma. Military Medical Sciences), pp. 188.

31. Zhang MW, Yu YX, Jin MJ, Pan YF, Jiang X, Li QL, Ma XY, Zhang SC, Chen K. Association of mir-605 and mir-149 genetic polymorphisms with related risk factors of lung cancer susceptibility. Jouranl of zhejiang University(Medical sciences). 2011; 40:265-271.

32. Liu Z, Li G, Wei S, Niu J, El-Naggar AK, Sturgis EM, Wei Q. Genetic variants in selected pre-microRNA genes and the risk of squamous cell carcinoma of the head and neck. Cancer. 2010; 116:4753-4760.

33. Tian T, Shu Y, Chen J, Hu Z, Xu L, Jin G, Liang J, Liu P, Zhou X, Miao R, Ma H, Chen Y, Shen H. A functional genetic variant in microRNA-196a2 is associated with increased susceptibility of lung cancer in Chinese. Cancer epidemiology, biomarkers and prevention: a publication of the American Association for Cancer Research, cosponsored by the American Society of Preventive Oncology. 2009; 18:1183-1187.

34. Wang ZW. WRN, BLM, BRCA1 and miRNAs Gene Polymorphism and the Susceptibility of Breast Cancer. Nanjing Medical University). 2009; 95.

35. Ma JY, Yan HJ, Yang ZH, Gu W. Rs895819 within miR-27a might be involved in development of non small cell lung 
cancer in the Chinese Han population. Asian Pacific journal of cancer prevention: APJCP. 2015; 16:1939-1944.

36. Qi P, Wang L, Zhou B, Yao WJ, Xu S, Zhou Y, Xie ZB. Associations of miRNA polymorphisms and expression levels with breast cancer risk in the Chinese population. Genet Mol Res. 2015; 14:6289-6296.

37. Yin Z, Cui Z, Guan P, Li X, Wu W, Ren Y, He Q, Zhou B. Interaction between Polymorphisms in Pre-MiRNA Genes and Cooking Oil Fume Exposure on the Risk of Lung Cancer in Chinese Non-Smoking Female Population. PloS one. 2015; 10:e128572.

38. Cao Y, Hu J, Fang Y, Chen Q, Li H. Association between a functional variant in microRNA-27a and susceptibility to colorectal cancer in a Chinese Han population. Genet Mol Res. 2014; 13:7420-7427.

39. Kupcinskas J, Bruzaite I, Juzenas S, Gyvyte U, Jonaitis L, Kiudelis G, Skieceviciene J, Leja M, Pauzas H, Tamelis A, Pavalkis D, Kupcinskas L. Lack of association between miR-27a, miR-146a, miR-196a-2, miR-492 and miR-608 gene polymorphisms and colorectal cancer. Scientific reports. 2014; 4:5993.

40. Kupcinskas J, Wex T, Link A, Leja M, Bruzaite I, Steponaitiene R, Juzenas S, Gyvyte U, Ivanauskas A, Ancans G, Petrenkiene V, Skieceviciene J, Kupcinskas L, Malfertheiner P. Gene polymorphisms of micrornas in Helicobacter pylori-induced high risk atrophic gastritis and gastric cancer. PloS one. 2014; 9:e87467.

41. Song B, Yan G, Hao H, Yang B. rs11671784 G/A and rs895819 A/G polymorphisms inversely affect gastric cancer susceptibility and miR-27a expression in a Chinese population. Medical science monitor. 2014; 20:2318-2326.

42. Wang Z, Sun X, Wang Y, Liu X, Xuan Y, Hu S. Association between miR-27a genetic variants and susceptibility to colorectal cancer. Diagnostic pathology. 2014; 9:146.

43. Zhang J, Huang X, Xiao J, Yang Y, Zhou Y, Wang X, Liu Q, Yang J, Wang M, Qiu L, Zheng Y, Zhang P, Li J, et al. Pri-miR-124 rs531564 and pri-miR-34b/c rs4938723 polymorphisms are associated with decreased risk of esophageal squamous cell carcinoma in Chinese populations. PloS one. 2014; 9: e100055.

44. Zhang N, Huo Q, Wang X, Chen X, Long L, Jiang L, Ma T, Yang Q. A genetic variant in pre-miR-27a is associated with a reduced breast cancer risk in younger Chinese population. Gene. 2013; 529:125-130.

45. Catucci I, Verderio P, Pizzamiglio S, Bernard L, Dall'olio V, Sardella D, Ravagnani F, Galastri L, Barile M, Peissel B, Zaffaroni D, Manoukian S, Radice P, et al. The SNP rs895819 in miR-27a is not associated with familial breast cancer risk in Italians. Breast cancer research and treatment. 2012; 133:805-807.

46. Hezova R, Kovarikova A, Bienertova-Vasku J, Sachlova M, Redova M, Vasku A, Svoboda M, Radova L, Kiss I, Vyzula R, Slaby O. Evaluation of SNPs in miR-196-a2, miR-27a and miR-146a as risk factors of colorectal cancer. World journal of gastroenterology: WJG. 2012; 18:2827-2831.
47. Shi D, Li P, Ma L, Zhong D, Chu H, Yan F, Lv Q, Qin C, Wang W, Wang M, Tong N, Zhang Z, Yin C. A genetic variant in pre-miR-27a is associated with a reduced renal cell cancer risk in a Chinese population. PloS one. 2012; 7:e46566.

48. Zhang MW. Study on the associations of lifestyle-related factors, genetic variants in miRNA encoding regions and miRNA binding sites with colorectal cancer risk. Zhejiang University). 2012; 150.

49. Zhou Y, Du WD, Chen G, Ruan J, Xu S, Zhou FS, Zuo XB, Lv ZJ, Zhang XJ. Association analysis of genetic variants in microRNA networks and gastric cancer risk in a Chinese Han population. Journal of cancer research and clinical oncology. 2012; 138:939-945.

50. Zhang P. Polymorphisms of micro RNA and ESR1 genes and their association with triple negative breast caneer risk and prognosis. Peking Union Medical College). 2011; 74.

51. Sun Q, Gu H, Zeng Y, Xia Y, Wang Y, Jing Y, Yang L, Wang B. Hsa-mir-27a genetic variant contributes to gastric cancer susceptibility through affecting miR-27a and target gene expression. Cancer science. 2010; 101:2241-2247.

52. Kontorovich T, Levy A, Korostishevsky M, Nir U, Friedman E. Single nucleotide polymorphisms in miRNA binding sites and miRNA genes as breast/ovarian cancer risk modifiers in Jewish high-risk women. International journal of cancer. 2010; 127:589-597.

53. Yang R, Schlehe B, Hemminki K, Sutter C, Bugert P, Wappenschmidt B, Volkmann J, Varon R, Weber BH, Niederacher D, Arnold N, Meindl A, Bartram CR, et al. A genetic variant in the pre-miR-27a oncogene is associated with a reduced familial breast cancer risk. Breast cancer research and treatment. 2010; 121:693-702.

54. He B, Pan Y, Cho WC, Xu Y, Gu L, Nie Z, Chen L, Song G, Gao T, Li R, Wang S. The association between four genetic variants in microRNAs (rs11614913, rs2910164, rs3746444, rs2292832) and cancer risk: evidence from published studies. PloS one. 2012; 7:e49032.

55. Zhang J, Liu YF, Gan Y. Lack of association between miR-149 C > T polymorphism and cancer susceptibility: a meta-analysis based on 4,677 cases and 4,830 controls. Molecular biology reports. 2012; 39:8749-8753.

56. Sevignani C, Calin GA, Nnadi SC, Shimizu M, Davuluri RV, Hyslop T, Demant P, Croce CM, Siracusa LD. MicroRNA genes are frequently located near mouse cancer susceptibility loci. Proceedings of the National Academy of Sciences of the United States of America. 2007; 104:8017-8022.

57. Guttilla IK, White BA. Coordinate regulation of FOXO1 by miR-27a, miR-96, and miR-182 in breast cancer cells. The Journal of biological chemistry. 2009; 284:23204-23216.

58. Ma Y, Yu S, Zhao W, Lu Z, Chen J. miR-27a regulates the growth, colony formation and migration of pancreatic cancer cells by targeting Sprouty2. Cancer letters. 2010; 298:150-158.

59. Zhang Z, Liu S, Shi R, Zhao G. miR-27 promotes human gastric cancer cell metastasis by inducing 
epithelial-to-mesenchymal transition. Cancer genetics. 2011; 204:486-491.

60. Zhou L, Yin B, Liu Y, Hong Y, Zhang C, Fan J. Mechanism and function of decreased FOXO1 in renal cell carcinoma. Journal of surgical oncology. 2012; 105:841-847.

61. Fletcher CE, Dart DA, Sita-Lumsden A, Cheng H, Rennie PS, Bevan CL. Androgen-regulated processing of the oncomir miR-27a, which targets Prohibitin in prostate cancer. Human molecular genetics. 2012; 21:3112-3127.

62. Parpart S, Wang XW. microRNA regulation and its consequences in cancer. Current pathobiology reports. $2013 ; 1: 71-79$.

63. Hayes J, Peruzzi PP, Lawler S. MicroRNAs in cancer: biomarkers, functions and therapy. Trends in molecular medicine. 2014; 20:460-469.

64. Wang Z, Lai J, Wang Y, Nie W, Guan X. The Hsa-miR-27a rs895819 (A > G) polymorphism and cancer susceptibility. Gene. 2013; 521:87-90.
65. Zhong S, Chen Z, Xu J, Li W, Zhao J. Pre-mir-27a rs895819 polymorphism and cancer risk: a meta-analysis. Molecular biology reports. 2013; 40:3181-3186.

66. Moher D, Liberati A, Tetzlaff J, Altman DG. Preferred reporting items for systematic reviews and meta-analyses: the PRISMA statement. Annals of internal medicine. 2009; 151:264-269.

67. Wells G, Shea B, O'connell D, Peterson J, Welch V, Losos M, Tugwell P. (2011). The Newcastle-Ottawa Scale (NOS) for assessing the quality of nonrandomised studies in meta-analyses.

68. Cochran WG. The combination of estimates from different experiments. Biometrics. 1954; 10:101-129.

69. Mantel N, Haenszel W. Statistical aspects of the analysis of data from retrospective studies of disease. Journal of the National Cancer Institute. 1959; 22:719-748.

70. DerSimonian R, Laird N. Meta-analysis in clinical trials. Controlled clinical trials. 1986; 7:177-188. 